\title{
THE OPEN DIHYPERGRAPH DICHOTOMY AND THE SECOND LEVEL OF THE BOREL HIERARCHY
}

\author{
RAPHAËL CARROY, BENJAMIN D. MILLER, AND DÁNIEL T. SOUKUP
}

\begin{abstract}
We show that several dichotomy theorems concerning the second level of the Borel hierarchy are special cases of the $\aleph_{0}$-dimensional generalization of the open graph dichotomy, which itself follows from the usual proof(s) of the perfect set theorem. Under the axiom of determinacy, we obtain the generalizations of these results from analytic metric spaces to separable metric spaces. We also consider connections between cardinal invariants and the chromatic numbers of the corresponding dihypergraphs.
\end{abstract}

\section{INTRODUCTION}

Basic notions. A topological space $X$ is Polish if it is second countable and completely metrizable. A topological space $X$ is analytic if it is a continuous image of a closed subset of $\mathbb{N}^{\mathbb{N}}$.

A subset of a topological space is $K_{\sigma}$ if it is a countable union of compact sets, $F_{\sigma}$ if it is a countable union of closed sets, $G_{\delta}$ if it is a countable intersection of open sets, $\Delta_{2}^{0}$ if it is both $F_{\sigma}$ and $G_{\delta}$, and Borel if it is in the smallest $\sigma$-algebra containing the open sets.

A function $\pi: X \rightarrow Y$ is $\Gamma$-measurable if the pre-image of every open set is in $\Gamma$. When $\Gamma$ is the family of Borel sets, we say that such a function is Borel. We say that a function $\pi: X \rightarrow Y$ is $\sigma$-continuous with $\Gamma$ witnesses if $X$ is the union of countably-many sets in $\Gamma$ on which $\phi$ is continuous.

Given a set $C \subseteq X$, we use $\sim C$ to denote the complement $C$ within $X$. We say that $C$ separates a set $A \subseteq X$ from a set $B \subseteq X$ if $A \subseteq C$ and $B \subseteq \sim C$. A subset of $\prod_{d \in D} X_{d}$ is a hyperrectangle if it is of the form $\prod_{d \in D} Y_{d}$, where $Y_{d} \subseteq X_{d}$ for all $d \in D$. When $D=2$, we say that such a set is a rectangle. The box topology on a product $\prod_{d \in D} X_{d}$ of topological spaces is the topology generated by the sets of the form $\prod_{d \in D} U_{d}$, where $U_{d} \subseteq X_{d}$ is open for all $d \in D$.

2010 Mathematics Subject Classification. Primary 03E15, 26A21, 28A05, 54H05. Key words and phrases. Coloring, dichotomy, separation, sigma-continuous.

The authors were supported in part by FWF Grants P28153, P29999, and I1921. 
A D-ary relation on $X$ is a subset of $X^{D}$. A homomorphism from a $D$-ary relation $R$ on $X$ to a $D$-ary relation $S$ on $Y$ is a map $\pi: X \rightarrow Y$ such that $\pi^{D}(R) \subseteq S$. A $D$-dimensional dihypergraph on $X$ is a $D$-ary relation $H$ on $X$ disjoint from the set $\triangle^{D}(X)$ of constant sequences. When $D=2$, we say that a symmetric such set is a graph. The complete D-dimensional dihypergraph on $X$ is the complement of $\triangle^{D}(X)$. A set $Y \subseteq X$ is $H$-independent if $H \uparrow Y=\emptyset$. A $\kappa$-coloring of a $D$-dimensional dihypergraph $H$ is a homomorphism from $H$ to the complete $D$-dimensional dihypergraph on a set of cardinality $\kappa$. The chromatic number of $H$, or $\chi(H)$, is the least cardinal $\kappa$ for which there is a $\kappa$-coloring of $H$. We say that an $\mathbb{N}$-dimensional dihypergraph is hereditary if it is closed under subsequences.

For each partial function $t: \mathbb{N} \rightarrow D$, set $\mathcal{N}_{t}=\left\{\mathbf{d} \in D^{\mathbb{N}} \mid t \sqsubseteq \mathbf{d}\right\}$. We use $(d),(d)^{n}$, and $(d)^{\infty}$ to denote the sequences with constant value $d$ in $D^{1}, D^{n}$, and $D^{\mathbb{N}}$. We use $s \frown t$ and $\bigoplus_{n \in N} s_{n}$ to denote concatenation.

We use $\mathbb{K}_{2^{\mathbb{N}}}$ to denote the complete graph on $2^{\mathbb{N}}$. More generally, we use $\mathbb{H}_{D^{\mathbb{N}}}$ to denote the $D$-dimensional dihypergraph on $D^{\mathbb{N}}$ given by $\mathbb{H}_{D^{\mathbb{N}}}=\bigcup_{t \in D<\mathbb{N}} \prod_{d \in D} \mathcal{N}_{t \frown(d)}$, and $\mathbb{H}_{D^{\mathbb{N}}}^{\prime}$ to denote the $D$-dimensional dihypergraph on $D^{\mathbb{N}}$ given by $\mathbb{H}_{D^{\mathbb{N}}}^{\prime}=\bigcup_{(\mathbf{d}, t) \in(D)^{D} \times D^{<\mathbb{N}}} \prod_{d \in D} \mathcal{N}_{t \frown(\mathbf{d}(d))}$, where $(D)^{N}$ denotes the set of injective elements of $D^{N}$.

The covering number of an ideal $\mathcal{I}$ on a set $X$ is the least cardinal $\operatorname{cov}(\mathcal{I})$ for which $X$ is the union of $\operatorname{cov}(\mathcal{I})$-many sets in $\mathcal{I}$. The cofinality of an ideal $\mathcal{I}$ on a set $X$ is the least cardinal $\operatorname{cof}(\mathcal{I})$ for which there is a set $\mathcal{J} \subseteq \mathcal{I}$ of cardinality $\operatorname{cof}(\mathcal{I})$ such that $\forall I \in \mathcal{I} \exists J \in \mathcal{J} I \subseteq J$. We use $\mathcal{M}$ to denote the $\sigma$-ideal on $\mathbb{R}$ consisting of the meager sets, and $\mathcal{N}$ to denote the $\sigma$-ideal on $[0,1]$ consisting of the Lebesgue null sets.

We write $\forall^{\infty} n \in N \phi(n)$ to indicate that $\{n \in N \mid \neg \phi(n)\}$ is finite. The order of eventual domination is the quasi-order on $\mathbb{N}^{\mathbb{N}}$ given by $\mathbf{c} \leq^{*} \mathbf{d} \Longleftrightarrow \forall^{\infty} n \in \mathbb{N} \mathbf{c}(n) \leq \mathbf{d}(n)$. The bounding number is the least cardinal $\mathfrak{b}$ for which there is a set $\mathcal{F} \subseteq \mathbb{N}^{\mathbb{N}}$ of cardinality $\mathfrak{b}$ such that $\forall \mathbf{c} \in \mathbb{N}^{\mathbb{N}} \exists \mathbf{d} \in \mathcal{F} \mathbf{d} \mathbb{Z}^{*} \mathbf{c}$. The dominating number is the least cardinal $\mathfrak{d}$ for which there is a set $\mathcal{F} \subseteq \mathbb{N}^{\mathbb{N}}$ of cardinality $\mathfrak{d}$ such that $\forall \mathbf{c} \in \mathbb{N}^{\mathbb{N}} \exists \mathbf{d} \in \mathcal{F} \mathbf{c} \leq^{*} \mathbf{d}$.

Motivation. In [Hur28], the existence of a minimal Borel subset of a Polish space that is not $F_{\sigma}$ was established. In [Wad12], this was generalized to every level of the Borel hierarchy under Borel determinacy. In [LSR87], these results were established in a far weaker subsystem of ZF, leading to parametrized generalizations. In [Lec13], they were further generalized to subsets of products.

In [LZ14], the question as to the circumstances under which a Borel subset of a product of two Polish spaces can be separated from 
another subset by a countable union of rectangles of low complexity was explored, as was the related question concerning the existence of a minimal Borel graph on a Polish space with no $\aleph_{0}$-coloring of low complexity. Positive results were obtained at the second level of the Borel hierarchy by adapting a classical proof of Hurewicz's original dichotomy theorem which, despite its simplicity, nevertheless relies on a finite injury argument, and does not yield the theorem in its natural generality. Moreover, the resulting minimal objects in some sense fail to be canonical, in that they depend on an arbitrary parameter. Positive results were also obtained at the third level of the Borel hierarchy, although the underlying arguments were quite intricate, as were the corresponding minimal objects. The question as to whether such results hold at the fourth level and beyond remains open.

In [JR82], it was shown that a function from an analytic metric space to a separable metric space is $G_{\delta^{-}}$measurable if and only if it is $\sigma$-continuous with closed witnesses. In [Sol98], this was shown to be a consequence of the fact that there is a two-element basis, consisting of non- $G_{\delta}$-measurable functions, for the family of Baire-class-one functions that are not $\sigma$-continuous with closed witnesses. As a corollary, it was also shown that if a Baire-class-one function is not $\sigma$-continuous with closed witnesses, then $\mathfrak{d}$ is the least cardinal $\kappa$ for which its domain is the union of $\kappa$-many closed sets on which the function is continuous. These results were established using ad-hoc recursive constructions reminiscent of those behind the level-two Lecomte-Zeleny results. Despite having received quite a bit of attention, the question as to whether these results generalize in their most natural form to higher levels of the Borel hierarchy remains open.

Here we show that a common generalization of the Kechris-LouveauWoodin generalization of Hurewicz's original dichotomy theorem (see [KLW87]) and the level-two results of Lecomte-Zeleny is a special case of the simplest descriptive set-theoretic dichotomy theorem, as is the Jayne-Rogers theorem. In addition to providing a simple unified explanation of these phenomena and canonical minimal objects, this observation also provides generalizations from analytic to separable metric spaces under the axiom of determinacy, as well as the generalization of Solecki's result concerning $\mathfrak{d}$ to Borel functions.

In a future paper, we will use the particular structure of the minimal objects so obtained to generalize Solecki's basis theorem to all Borel functions, while simultaneously strengthening it using a finer notion of embeddability. In another future paper, we will show that the generalizations of Hurewicz's theorem to every level of the difference hierarchy at the second level of the Borel hierarchy (including the dichotomy 
theorem established in [SR78] characterizing the circumstances under which a Borel set is a difference of two $F_{\sigma}$ sets) are also special cases of the simplest descriptive set-theoretic dichotomy theorem. While our hope is that this approach will lead to unified common generalizations to all levels of the Borel hierarchy, the question as to whether such arguments can even be pushed to the third level remains open.

The underlying dichotomy. The perfect set theorem for a class $\Gamma$ of topological spaces is the statement that for all $X \in \Gamma$, either $|X| \leq \aleph_{0}$ or there is a continuous injection of $2^{\mathbb{N}}$ into $X$. In [Sou17], this simplest of descriptive set-theoretic dichotomy theorems was established for analytic Hausdorff spaces. In [Dav64], it was generalized to subsets of such spaces under the axiom of determinacy.

The open graph dichotomy for a class $\Gamma$ of topological spaces is the statement that for all $X \in \Gamma$ and open graphs $G$ on $X$, either $\chi(G) \leq \aleph_{0}$ or there is a continuous homomorphism from $\mathbb{K}_{2^{\mathbb{N}}}$ to $G$. In [Fen93], this generalization of the perfect set theorem was established for analytic Hausdorff spaces, as was its generalization to subsets of such spaces under the axiom of determinacy. This was achieved by showing that the usual proofs of the perfect set theorem also yield the open graph dichotomy, giving a sense in which the latter is also among the simplest descriptive set-theoretic dichotomy theorems.

The box-open D-dimensional dihypergraph dichotomy for a class $\Gamma$ of topological spaces, or $\operatorname{OGD}^{D}(\Gamma)$, is the statement that for all $X \in \Gamma$ and box-open $D$-dimensional dihypergraphs $H$ on $X$, either $\chi(H) \leq \aleph_{0}$ or there is a continuous homomorphism from $\mathbb{H}_{D^{\mathbb{N}}}$ to $H$. In $\S 1$, we establish this generalization of the open graph dichotomy for analytic Hausdorff spaces, as well as its generalization to subsets of such spaces under the axiom of determinacy. As in [Fen93], we achieve this by showing that the usual proofs of the perfect set theorem easily adapt to yield the open dihypergraph dichotomy, giving a sense in which even the latter is among the simplest descriptive set-theoretic dichotomy theorems as well.

Applications. In $\S 2$, we give a first glimpse into how topological properties can be codified into dihypergraphs by showing that the KechrisSaint Raymond generalizations of Hurewicz's characterization of the circumstances under which a Polish space is $K_{\sigma}$ (see [Kec77] or [SR75]) is a special case of the box-open dihypergraph dichotomy.

In $\S 3$, we establish the basic properties of partial compactifications of $\mathbb{N}^{\mathbb{N}}$ arising from the box-open dihypergraph dichotomy.

In $\S 4$, we show that a special case of the open dihypergraph dichotomy yields a characterization of the circumstances under which an 
analytic subset of a $D$-fold product of metric spaces can be separated from another subset by a countable union of closed hyperrectangles. As a corollary, we obtain a characterization of the circumstances under which a $D$-dimensional dihypergraph on an analytic metric space has a $\boldsymbol{\Delta}_{2}^{0}$-measurable $\aleph_{0}$-coloring. We also obtain the generalizations in which analyticity is weakened to separability under the axiom of determinacy. The Kechris-Louveau-Woodin and level-two Lecomte-Zeleny theorems are straightforward consequences of these results.

In $\S 5$, we show that a special case of the box-open dihypergraph dichotomy yields the Jayne-Rogers characterization of the circumstances under which a function from an analytic metric space to a separable metric space is $\sigma$-continuous with closed witnesses. We also obtain the generalization in which analyticity is weakened to separability under the axiom of determinacy.

In $\S 6$, we note that $\chi\left(\mathbb{H}_{\mathbb{N}^{\mathbb{N}}}\right)=\operatorname{cov}(\mathcal{M})$ and $\chi\left(\mathbb{H}_{\mathbb{N}^{\mathbb{N}}}^{\prime}\right)=\mathfrak{d}$. In conjunction with the box-open dihypergraph dichotomy, the latter fact easily yields the promised generalization of Solecki's theorem. We also show that if $2<D<\aleph_{0}$, then $\chi\left(\mathbb{H}_{D^{\mathbb{N}}}\right)$ is at least $\mathfrak{b} \cdot \operatorname{cov}(\mathcal{N})$, consistently strictly below $\mathfrak{d}$, and consistently strictly above $\operatorname{cof}(\mathcal{N})$.

We work in the base theory ZF + DC throughout, with the exception of the final section, where we work in ZFC so as to keep our language as transparent as possible.

\section{THE BOX-OPEN DIHYPERGRAPH DICHOTOMY}

Despite the fact that it is essentially the same as the usual proof of the perfect set theorem for analytic Hausdorff spaces, we provide the proof of the analogous instance of the open dihypergraph dichotomy for the sake of the reader.

Theorem 1.1. Suppose that $D$ is a discrete space of cardinality at least two, $X$ is an analytic Hausdorff space, and $H$ is a box-open $D$ dimensional dihypergraph on $X$. Then exactly one of the following holds:

(1) There is an $\aleph_{0}$-coloring of $H$.

(2) There is a continuous homomorphism from $\mathbb{H}_{D^{\mathbb{N}}}$ to $H$.

Proof. To see that the two conditions are mutually exclusive, it is sufficient to show that there is no $\aleph_{0^{-c o l o r i n g}}$ of $\mathbb{H}_{D^{\mathbb{N}}}$. Towards this end, suppose that $X \subseteq D^{\mathbb{N}}$ and $c: X \rightarrow \mathbb{N}$ is an $\aleph_{0}$-coloring of $\mathbb{H}_{D^{\mathbb{N}}} \uparrow X$, recursively find $d_{n} \in D$ such that $n \notin c\left(\mathcal{N}_{\left(d_{m}\right)_{m \leq n}}\right)$ for all $n \in \mathbb{N}$, and observe that $\left(d_{n}\right)_{n \in \mathbb{N}} \notin c^{-1}(\mathbb{N})$, thus $X \neq D^{\mathbb{N}}$. 
To see that at least one of the conditions hold, we can assume that $X \neq \emptyset$, in which case there is a continuous surjection $\pi: \mathbb{N}^{\mathbb{N}} \rightarrow X$. By replacing $H$ with its pullback through $\pi$, we can assume that $X=\mathbb{N}^{\mathbb{N}}$.

Set $S=\left\{s \in \mathbb{N}<\mathbb{N} \mid H \uparrow \mathcal{N}_{s}\right.$ has an $\aleph_{0}$-coloring $\}$ and $Y=\sim \bigcup_{s \in S} \mathcal{N}_{s}$. Note that if $s \in \sim S$, then there is no $\aleph_{0}$-coloring of $H \uparrow\left(\mathcal{N}_{s} \cap Y\right)$, so there exists $\left(y_{d}\right)_{d \in D} \in H \uparrow\left(\mathcal{N}_{s} \cap Y\right)$, thus the fact that $H$ is box open yields a sequence $\left(s_{d}\right)_{d \in D} \in(\sim S)^{D}$ of proper extensions of $s$ such that $\prod_{d \in D} \mathcal{N}_{s_{d}} \subseteq H$. It follows that if there is no $\aleph_{0}$-coloring of $H$, then there is a function $f: D^{<\mathbb{N}} \rightarrow \sim S$ such that:

(a) $\forall d \in D \forall t \in D^{<\mathbb{N}} f(t) \sqsubset f(t \frown(d))$.

(b) $\forall t \in D^{<\mathbb{N}} \prod_{d \in D} \mathcal{N}_{f(t \frown(d))} \subseteq H$.

Condition (a) ensures that we obtain a continuous function $\phi: D^{\mathbb{N}} \rightarrow Y$ by setting $\phi(\mathbf{d})=\bigcup_{n \in \mathbb{N}} f(\mathbf{d}\lceil n)$, and condition (b) implies that $\phi$ is a homomorphism from $\mathbb{H}_{D^{\mathbb{N}}}$ to $H$.

As in [Fen93], the same argument yields the natural generalization to $\kappa$-Souslin Hausdorff spaces, and a derivative can be used to avoid the need for copious amounts of choice. However, in order to establish the open dihypergraph dichotomy for all subsets of analytic metric spaces under the axiom of determinacy, the natural analog of the game considered in [Fen93, §3] is insufficient, as it requires the first player to play $|D|$-many natural numbers in each round. We next show that a slowed-down version of this game can be used instead.

Given a box-open $\mathbb{N}$-dimensional dihypergraph $H$ on $\mathbb{N}^{\mathbb{N}}$ and a set $X \subseteq \mathbb{N}^{\mathbb{N}}$, consider the $\omega$-length two-player game whose $n^{\text {th }}$ round consists of the first player playing a sequence $s_{n} \in \mathbb{N}<\mathbb{N}$ with the property that $i_{m}=1 \Longrightarrow s_{m} \sqsubset s_{n}$ for all $m<n$, and then the second playing a natural number $i_{n}<2$. There are two types of runs of the game, depending on whether the set $N=\left\{n \in \mathbb{N} \mid i_{n}=1\right\}$ is finite or infinite. In the former case, the first player wins if and only if $\prod_{n \in \mathbb{N}} \mathcal{N}_{s_{m+n}} \subseteq H$, where $m$ is the least natural number strictly larger than every element of $N$. In the latter, the first player wins if and only if $\bigcup_{n \in N} s_{n} \in X$.

Proposition 1.2. Suppose that $H$ is a box-open $\mathbb{N}$-dimensional dihypergraph on $\mathbb{N}^{\mathbb{N}}$ and $X \subseteq \mathbb{N}^{\mathbb{N}}$.

(1) The first player has a winning strategy if and only if there is a continuous homomorphism from $\mathbb{H}_{\mathbb{N}^{\mathbb{N}}}$ to $H \uparrow X$.

(2) The second player has a winning strategy if and only if there is an $\aleph_{0}$-coloring of $H \uparrow X$.

Proof. Let $S$ denote the set of sequences $s \in 2^{<\mathbb{N}}$ that do not end in zero. Let $[\mathbb{N}]^{\mathbb{N}}$ denote the set of strictly increasing elements of $\mathbb{N}^{\mathbb{N}}$, define $\pi_{\mathbb{N}^{\mathbb{N}},[\mathbb{N}]^{\mathbb{N}}}: \mathbb{N}^{\mathbb{N}} \rightarrow[\mathbb{N}]^{\mathbb{N}}$ by $\pi_{\mathbb{N}^{\mathbb{N}},[\mathbb{N}]^{\mathbb{N}}}(b)(n)=n+\sum_{m \leq n} b(m)$, and 
define $\pi_{[\mathbb{N}]^{\mathbb{N}}, 2^{\mathbb{N}}}:[\mathbb{N}]^{\mathbb{N}} \rightarrow 2^{\mathbb{N}}$ by $\pi_{[\mathbb{N}]^{\mathbb{N}}, 2^{\mathbb{N}}}(b)=\mathbf{1}_{b(\mathbb{N})}$. Then the function $\pi_{\mathbb{N}^{\mathbb{N}}, 2^{\mathbb{N}}}=\pi_{\left[\mathbb{N}^{\mathbb{N}}, 2^{\mathbb{N}}\right.} \circ \pi_{\mathbb{N}^{\mathbb{N}},[\mathbb{N}]^{\mathbb{N}}}$ is a homeomorphism from $\mathbb{N}^{\mathbb{N}}$ to the space $C=\left\{c \in 2^{\mathbb{N}}|| \operatorname{supp} c \mid=\aleph_{0}\right\}$, in addition to being an isomorphism of $\mathbb{H}_{\mathbb{N}^{\mathbb{N}}}$ with the restriction of the box-open $\mathbb{N}$-dimensional dihypergraph $H_{2^{\mathbb{N}}}=\bigcup_{s \in S} \prod_{n \in \mathbb{N}} \mathcal{N}_{s \frown(0)^{n} \frown(1)}$ to $C$.

Suppose that $\tau$ is a winning strategy for the first player, and define $\phi: C \rightarrow \mathbb{N}^{\mathbb{N}}$ by $\phi(c)=\bigcup_{n \in \operatorname{supp} c} \tau(c \uparrow n)$. To see that $\phi$ is a homomorphism from $H_{2^{\mathbb{N}}} \uparrow C$ to $H$, note that if $\left(c_{n}\right)_{n \in \mathbb{N}} \in H_{2^{\mathbb{N}}} \uparrow C$, then there exists $s \in S$ for which $\left(c_{n}\right)_{n \in \mathbb{N}} \in \prod_{n \in \mathbb{N}} \mathcal{N}_{s \frown(0)^{n} \frown(1)}$, in which case $\left(\phi\left(c_{n}\right)\right)_{n \in \mathbb{N}} \in \prod_{n \in \mathbb{N}} \mathcal{N}_{\tau\left(s \wedge(0)^{n}\right)}$, so the fact that the first player wins runs of the game of the first type ensures that $\left(\phi\left(c_{n}\right)\right)_{n \in \mathbb{N}} \in H$. The fact that the first player wins runs of the game of the second type implies that $\phi(C) \subseteq X$.

Conversely, suppose that $\phi: C \rightarrow X$ is a continuous homomorphism from $H_{2^{\mathbb{N}}} \uparrow C$ to $H$. For each non-empty sequence $s \in S$, let $s^{-}$denote the immediate predecessor of $s$. We will recursively construct functions $\sigma: 2^{<\mathbb{N}} \rightarrow S$ and $\tau: 2^{<\mathbb{N}} \rightarrow \mathbb{N}<\mathbb{N}$ such that:

(a) $\forall s \in S \prod_{n \in \mathbb{N}} \mathcal{N}_{\tau\left(s \wedge(0)^{n}\right)} \subseteq H$.

(b) $\forall s \in 2^{<\mathbb{N}} \phi\left(\mathcal{N}_{\sigma(s)}\right) \subseteq \mathcal{N}_{\tau(s)}$.

(c) $\forall n \in \mathbb{N} \forall s \in S \backslash\{\emptyset\} \tau\left(s^{-}\right) \sqsubset \tau\left(s \frown(0)^{n}\right)$.

(d) $\forall n \in \mathbb{N} \forall s \in S \backslash\{\emptyset\} \sigma\left(s^{-}\right) \frown(0)^{n} \frown(1) \sqsubseteq \sigma\left(s \frown(0)^{n}\right)$.

Suppose that $s \in S$ and we have already found $\sigma(r)$ and $\tau(r)$ for all $r \sqsubset s$. If $s=\emptyset$, then set $u_{s}=v_{s}=\emptyset$, and otherwise define $u_{s}=\sigma\left(s^{-}\right)$and $v_{s}=\tau\left(s^{-}\right)$. As $\left(u_{s} \frown(0)^{n} \frown(1)^{\infty}\right)_{n \in \mathbb{N}} \in H_{2^{\mathbb{N}}} \uparrow C$, there are strict extensions $\tau\left(s \frown(0)^{n}\right) \sqsubset \phi\left(u_{s} \frown(0)^{n} \frown(1)^{\infty}\right)$ of $v_{s}$ such that $\prod_{n \in \mathbb{N}} \mathcal{N}_{\tau\left(s \frown(0)^{n}\right)} \subseteq H$, as well as positive integers $k_{n, s}$ such that $\phi\left(\mathcal{N}_{u_{s} \frown(0)^{n} \curvearrowleft(1)^{k_{n}, s}}\right) \subseteq \mathcal{N}_{\tau\left(s \frown(0)^{n}\right)}$ for all $n \in \mathbb{N}$. We complete the construction by setting $\sigma\left(s \frown(0)^{n}\right)=u_{s} \frown(0)^{n} \frown(1)^{k_{n, s}}$ for all $n \in \mathbb{N}$. To see that $\tau$ is a winning strategy for the first player, note that the first player wins runs of the game of the first type by condition (a), whereas the other conditions ensure that if $c \in C$, then $\phi\left(\bigcup_{n \in \operatorname{supp} c} \sigma(c\lceil n))=\bigcup_{n \in \operatorname{supp} c} \tau(c\lceil n)\right.$, thus the first player wins runs of the game of the second type.

Suppose now that $\tau$ is a winning strategy for the second player. Let $T$ denote the set of partial runs of the game against $\tau$ for which $(\tau(t \uparrow\{0, \ldots, n\}))_{n<|t|} \in S$, and associate with each $t \in T$ the set $X_{t}=\{x \in X \mid t \neq \emptyset \Longrightarrow t(|t|-1) \sqsubseteq x\}$. If $\left(x_{n}\right)_{n \in \mathbb{N}} \in H \uparrow X_{t}$, then there are sequences $t_{n} \sqsubset x_{n}$ with the property that $\prod_{n \in \mathbb{N}} \mathcal{N}_{t_{n}} \subseteq H$ and $t \neq \emptyset \Longrightarrow \forall n \in \mathbb{N} t(|t|-1) \sqsubset t_{n}$, so the fact that the second player wins runs of the game of the first type therefore yields $n \in \mathbb{N}$ for which $t \frown\left(t_{m}\right)_{m \leq n} \in T$ and $x_{n} \in X_{t \frown\left(t_{m}\right)_{m \leq n}}$. In particular, it follows 
that the sets of the form $X_{t} \backslash \bigcup_{t \sqsubset u, u \in T} X_{u}$ are $H$-independent. As the fact that the second player wins runs of the game of the second type ensures that every $x \in X$ appears in a set of the latter form, it follows that there is an $\aleph_{0}$-coloring of $H \uparrow X$.

Conversely, suppose that $c: X \rightarrow \mathbb{N}$ is an $\aleph_{0}$-coloring of $H \uparrow X$, and let $\tau$ be the strategy for the second player in which 0 is played in the $n^{\text {th }}$ round of the game if and only if $c^{-1}\left(\left\{k_{n}\right\}\right) \cap \mathcal{N}_{s_{n}} \neq \emptyset$, where $k_{n}=\left|\left\{m<n \mid i_{m}=1\right\}\right|$. The fact that the sets of the form $c^{-1}(\{k\})$ are $H$-independent ensures that the second player wins runs of the game of the first type, while the fact that $X \subseteq c^{-1}(\mathbb{N})$ implies that the second player wins runs of the game of the second type.

In particular, we obtain the following.

Theorem 1.3 (AD). Suppose that $Y$ is an analytic Hausdorff space, $H$ is a box-open $\mathbb{N}$-dimensional dihypergraph on $Y$, and $X \subseteq Y$. Then exactly one of the following holds:

(1) There is an $\aleph_{0}$-coloring of $H \uparrow X$.

(2) There is a continuous homomorphism from $\mathbb{H}_{\mathbb{N}^{\mathbb{N}}}$ to $H \uparrow X$.

Proof. As noted in the proof of Theorem 1.1, the two conditions are mutually exclusive, so it is sufficient to show that at least one of them holds. We can also assume that $Y \neq \emptyset$, in which case there is a continuous surjection $\phi: \mathbb{N}^{\mathbb{N}} \rightarrow Y$. By replacing $H$ and $X$ with their pullbacks through $\pi$, we can assume that $Y=\mathbb{N}^{\mathbb{N}}$. But Proposition 1.2 then ensures that one of the two conditions holds.

\section{2. $K_{\sigma}$ SETS}

Given a topological space $X$, let $H_{X}$ denote the $\mathbb{N}$-dimensional dihypergraph on $X$ consisting of all injective sequences $\left(x_{n}\right)_{n \in \mathbb{N}}$ of elements of $X$ with no convergent subsequence.

Proposition 2.1. Suppose that $X$ is a metric space.

(1) The dihypergraph $H_{X}$ is box open.

(2) There is an $\aleph_{0}$-coloring of the restriction of $H_{X}$ to a set $Y \subseteq X$ if and only if $Y$ is contained in a $K_{\sigma}$ subset of $X$.

(3) A continuous function $\phi: \mathbb{N}^{\mathbb{N}} \rightarrow X$ is a homomorphism from $\mathbb{H}_{\mathbb{N}^{\mathbb{N}}}$ to $H_{X}$ if and only if it is an injective closed map.

Proof. To see (1), note that if $\left(x_{n}\right)_{n \in \mathbb{N}} \in H_{X}$, then there exist positive real numbers $\epsilon_{n} \rightarrow 0$ such that $\rho_{X}\left(x_{m}, x_{n}\right) \geq 2 \epsilon_{n}$ for all natural numbers $m \neq n$, in which case $\prod_{n \in \mathbb{N}} \mathcal{B}_{X}\left(x_{n}, \epsilon_{n}\right) \subseteq H_{X}$.

To see (2), it is sufficient to observe that a set $Y \subseteq X$ is $H_{X^{-}}$ independent if and only if its closure is compact. 
To see (3), note first that if $\phi$ is an injective closed map, then the fact that each sequence $\left(\mathbf{d}_{n}\right)_{n \in \mathbb{N}} \in \mathbb{H}_{\mathbb{N}^{\mathbb{N}}}$ is an injective enumeration of a closed discrete set ensures that the same holds of $\left(\phi\left(\mathbf{d}_{n}\right)\right)_{n \in \mathbb{N}}$. Conversely, suppose that $\phi$ is a homomorphism from $\mathbb{H}_{\mathbb{N}^{\mathbb{N}}}$ to $H_{X}$. The fact that $H_{X}$ consists solely of injective sequences easily implies that $\phi$ is injective. To see that $\phi$ is a closed map, it is sufficient to show that every sequence $\left(\mathbf{d}_{n}\right)_{n \in \mathbb{N}}$ of elements of $\mathbb{N}^{\mathbb{N}}$ for which $\left(\phi\left(\mathbf{d}_{n}\right)\right)_{n \in \mathbb{N}}$ converges has a convergent subsequence. If there exists $\mathbf{d} \in \mathbb{N}^{\mathbb{N}}$ such that $\mathbf{d}_{n}(i)<\mathbf{d}(i)$ for all $i, n \in \mathbb{N}$, then the compactness of $\prod_{i \in \mathbb{N}} \mathbf{d}(i)$ yields the desired subsequence. So suppose, towards a contradiction, that there does not exist such a $\mathbf{d}$. Then there is a least $k \in \mathbb{N}$ for which $\left\{\mathbf{d}_{n}(k) \mid n \in \mathbb{N}\right\}$ is infinite. By passing to a subsequence, we can assume that for all distinct $m, n \in \mathbb{N}$, the sequences $\mathbf{d}_{m}$ and $\mathbf{d}_{n}$ differ from one another for the first time on their $k^{\text {th }}$ coordinates. By passing to a further subsequence, we can assume that $\left(\mathbf{d}_{n}\right)_{n \in \mathbb{N}}$ is a subsequence of an element of $\mathbb{H}_{\mathbb{N}^{\mathbb{N}}}$, so $\left(\phi\left(\mathbf{d}_{n}\right)\right)_{n \in \mathbb{N}}$ is a subsequence of an element of $H_{X}$, contradicting the fact that it converges.

In particular, we obtain the following.

Theorem $2.2\left(\operatorname{OGD}^{\mathbb{N}}(\Gamma)\right)$. Suppose that $X$ is a metric space and $Y \subseteq X$ is in $\Gamma$. Then exactly one of the following holds:

(1) The set $Y$ is contained in a $K_{\sigma}$ subset of $X$.

(2) There is a closed continuous injection $\phi: \mathbb{N}^{\mathbb{N}} \rightarrow X$ with the property that $\phi\left(\mathbb{N}^{\mathbb{N}}\right) \subseteq Y$.

Proof. This follows from Proposition 2.1.

叉

The special cases of Theorem 2.2 where $\Gamma$ is either the family of analytic spaces or the family of separable spaces easily yields the Kechris-Saint Raymond generalizations of Hurewicz's characterization of the circumstances under which a Polish space is $K_{\sigma}$.

\section{Partial COMpactifications}

For each topological space $X$ and discrete set $D \subseteq X$, endow the set $\operatorname{Ext}_{X}\left(D^{\mathbb{N}}\right)=D^{\mathbb{N}} \cup\left\{t \frown(x) \mid t \in D^{<\mathbb{N}}\right.$ and $\left.x \in \sim D\right\}$ with the topology generated by the sets $\mathcal{N}_{t, U}=\left\{\mathbf{d} \in \operatorname{Ext}_{X}\left(D^{\mathbb{N}}\right) \mid t \sqsubset \mathbf{d}\right.$ and $\left.\mathbf{d}(|t|) \in U\right\}$, where $t \in D^{<\mathbb{N}}$ and $U \subseteq X$ is open. Such spaces arise naturally in applications of the open dihypergraph dichotomy. An instructive example to consider is $\operatorname{Ext}_{\mathbb{N}_{*}}\left(\mathbb{N}^{\mathbb{N}}\right)$, where $\mathbb{N}_{*}=\mathbb{N} \cup\{\infty\}$ denotes the one-point compactification of $\mathbb{N}$.

Proposition 3.1. Suppose that $X$ is a compact space and $D \subseteq X$ is discrete and open. Then $\operatorname{Ext}_{X}\left(D^{\mathbb{N}}\right)$ is compact. 
Proof. Set $\mathcal{U}=\left\{\mathcal{N}_{t, U} \mid t \in D^{<\mathbb{N}}\right.$ and $U \subseteq X$ is open $\}$, and for each sequence $t \in D^{<\mathbb{N}}$ and family $\mathcal{V} \subseteq \mathcal{U}$, let $\mathcal{V}_{t}$ denote the family of open sets $V \subseteq X$ for which $\mathcal{N}_{t, V} \in \mathcal{V}$.

Lemma 3.2. Suppose that $t \in D^{<\mathbb{N}}$ and $\mathcal{V} \subseteq \mathcal{U}$ covers $\mathcal{N}_{t, X}$. If for all $d \in D$ there is a finite set $\mathcal{F}_{d} \subseteq \mathcal{V}$ covering $\mathcal{N}_{t \wedge(d), X}$, then there is a finite set $\mathcal{F} \subseteq \mathcal{V}$ covering $\mathcal{N}_{t, X}$.

Proof. We can assume that there do not exist $s \sqsubset t$ and an open set $U \subseteq X$ for which $t(|s|) \in U$ and $\mathcal{N}_{s, U} \in \mathcal{V}$. Then there is a finite set $\mathcal{F} \subseteq \mathcal{V}_{t}$ covering $\sim D$, in which case the set $F=\sim \bigcup \mathcal{F}$ is compact and contained in $D$, thus finite. But $\mathcal{F} \cup \bigcup_{d \in F} \mathcal{F}_{d}$ covers $\mathcal{N}_{t, X}$.

If $\mathcal{V} \subseteq \mathcal{U}$ is a cover of $\operatorname{Ext}_{X}\left(D^{\mathbb{N}}\right)$ with no finite subcover, then by recursively applying the contrapositive of Lemma 3.2, we obtain a sequence $\mathbf{d} \in D^{\mathbb{N}}$ such that for no $n \in \mathbb{N}$ is there a finite subset of $\mathcal{V}$ covering $\mathcal{N}_{\mathbf{d}\lceil n, X}$, contradicting the fact that $\mathbf{d} \in \bigcup \mathcal{V}$.

Proposition 3.3. Suppose that $X$ is a (complete) ultrametric space and $D \subseteq X$ is discrete and open. Then $\operatorname{Ext}_{X}\left(D^{\mathbb{N}}\right)$ has a (complete) compatible ultrametric.

Proof. By replacing $\rho_{X}$ with $\rho_{X} /\left(1+\rho_{X}\right)$, we can assume that $\rho_{X}<1$. Fix real numbers $\epsilon_{d}>0$ such that $\sup _{d \in D} \epsilon_{d}<1$ and $\mathcal{B}\left(d, \epsilon_{d}\right)=\{d\}$ for all $d \in D$, and define $\rho: \operatorname{Ext}_{X}\left(D^{\mathbb{N}}\right) \times \operatorname{Ext}_{X}\left(D^{\mathbb{N}}\right) \rightarrow \mathbb{R}$ by

$$
\rho(\mathbf{c}, \mathbf{d})= \begin{cases}0 & \text { if } \mathbf{c}=\mathbf{d} \text { and } \\ \rho_{X}(\mathbf{c}(n), \mathbf{d}(n)) \prod_{m<n} \epsilon_{\mathbf{c}(m)} & \text { otherwise, }\end{cases}
$$

where $n=n(\mathbf{c}, \mathbf{d})$ is the least natural number for which $\mathbf{c}(n) \neq \mathbf{d}(n)$.

To establish that $\rho$ is an ultrametric, it is sufficient to show that if $\mathbf{b}, \mathbf{c}, \mathbf{d} \in \operatorname{Ext}_{X}\left(D^{\mathbb{N}}\right)$ are distinct, then $\rho(\mathbf{b}, \mathbf{d}) \leq \max \{\rho(\mathbf{b}, \mathbf{c}), \rho(\mathbf{c}, \mathbf{d})\}$. Setting $n=\max \{n(\mathbf{b}, \mathbf{c}), n(\mathbf{c}, \mathbf{d})\}$, there are three cases to check:

(1) If $n(\mathbf{b}, \mathbf{d})<n$, then $\rho(\mathbf{b}, \mathbf{d}) \in\{\rho(\mathbf{b}, \mathbf{c}), \rho(\mathbf{c}, \mathbf{d})\}$.

(2) If $n(\mathbf{b}, \mathbf{d})=n$, then $n(\mathbf{b}, \mathbf{c})=n(\mathbf{b}, \mathbf{d})=n(\mathbf{c}, \mathbf{d})$, so

$$
\begin{aligned}
\rho(\mathbf{b}, \mathbf{d}) & =\rho_{X}(\mathbf{b}(n), \mathbf{d}(n)) \prod_{m<n} \epsilon_{\mathbf{b}(m)} \\
& \leq \max \left\{\rho_{X}(\mathbf{b}(n), \mathbf{c}(n)), \rho_{X}(\mathbf{c}(n), \mathbf{d}(n))\right\} \prod_{m<n} \epsilon_{\mathbf{b}(m)} \\
& =\max \{\rho(\mathbf{b}, \mathbf{d}), \rho(\mathbf{c}, \mathbf{d})\} .
\end{aligned}
$$

(3) If $n(\mathbf{b}, \mathbf{d})>n$, then $\rho(\mathbf{b}, \mathbf{d})<\prod_{m \leq n} \epsilon_{\mathbf{b}(m)} \leq \rho(\mathbf{b}, \mathbf{c})$.

To see that the topology generated by $\rho$ is contained in that of $\operatorname{Ext}_{X}\left(D^{\mathbb{N}}\right)$, suppose that $\mathbf{d} \in \operatorname{Ext}_{X}\left(D^{\mathbb{N}}\right)$ and $\epsilon>0$. If $\mathbf{d} \in D^{\mathbb{N}}$, then there exists $n \in \mathbb{N}$ sufficiently large that $\prod_{m<n} \epsilon_{\mathbf{d}(m)} \leq \epsilon$, in which case $\mathcal{N}_{\mathbf{d} \mid n, X}$ is an open neighborhood of $\mathbf{d}$ contained in $\mathcal{B}(\mathbf{d}, \epsilon)$. Otherwise, 
there exist $t \in D^{<\mathbb{N}}$ and $x \in \sim D$ for which $\mathbf{d}=t \frown(x)$, in which case $\mathcal{N}_{t, \mathcal{B}_{X}(x, \epsilon)}$ is an open neighborhood of $\mathbf{d}$ contained in $\mathcal{B}(\mathbf{d}, \epsilon)$.

To see that the topology of $\operatorname{Ext}_{X}\left(D^{\mathbb{N}}\right)$ is contained in that generated by $\rho$, suppose that $t \in D^{<\mathbb{N}}, U \subseteq X$ is open, and $\mathbf{d} \in \mathcal{N}_{t, U}$, and fix $0<\epsilon<1$ such that $\mathcal{B}_{X}(\mathbf{d}(|t|), \epsilon) \subseteq U$. Then $\mathcal{B}\left(\mathbf{d}, \epsilon \prod_{n<|t|} \epsilon_{t(n)}\right) \subseteq \mathcal{N}_{t, U}$.

To see that the completeness of $\rho_{X}$ yields that of $\rho$, suppose that $\left(\mathbf{d}_{k}\right)_{k \in \mathbb{N}}$ is an injective Cauchy sequence of elements of $\operatorname{Ext}_{X}\left(D^{\mathbb{N}}\right)$. If there exists $\mathbf{d} \in D^{\mathbb{N}}$ with the property that $\mathbf{d}_{k}(n) \rightarrow \mathbf{d}(n)$ for all $n \in \mathbb{N}$, then $\mathbf{d}_{k} \rightarrow \mathbf{d}$. Otherwise, there is a sequence $t \in D^{<\mathbb{N}}$ of maximal length such that $\mathbf{d}_{k}(n) \rightarrow t(n)$ for all $n<|t|$. By passing to a subsequence of $\left(\mathbf{d}_{k}\right)_{k \in \mathbb{N}}$, we can assume that $n\left(\mathbf{d}_{j}, \mathbf{d}_{k}\right)=|t|$, thus $\rho\left(\mathbf{d}_{j}, \mathbf{d}_{k}\right)=\rho_{X}\left(\mathbf{d}_{j}(|t|), \mathbf{d}_{k}(|t|)\right) \prod_{n<|t|} \epsilon_{t(n)}$, for all distinct $j, k \in \mathbb{N}$. It follows that $\left(\mathbf{d}_{k}(|t|)\right)_{k \in \mathbb{N}}$ is Cauchy, and therefore convergent, thus $\left(\mathbf{d}_{k}\right)_{k \in \mathbb{N}}$ is convergent as well.

Let $\operatorname{Cnvg}(X)$ denote the set of convergent sequences $\left(x_{n}\right)_{n \in \mathbb{N}}$ of elements of $X$, and let $\operatorname{Cnvg}_{X}\left(D^{\mathbb{N}}\right)$ denote the $\mathbb{N}$-ary relation on $D^{\mathbb{N}}$ given by $\operatorname{Cnvg}_{X}\left(D^{\mathbb{N}}\right)=\bigcup_{(\mathbf{d}, t) \in\left(\operatorname{Cnvg}(X) \cap D^{\mathbb{N}}\right) \times D^{<\mathbb{N}}} \prod_{n \in \mathbb{N}} \mathcal{N}_{t \frown(\mathbf{d}(n))}$. In our applications of the open dihypergraph dichotomy, the following fact will yield extensions of homomorphisms between dihypergraphs.

Proposition 3.4. Suppose that $X$ and $Y$ are metric spaces, $D \subseteq X$ is dense, discrete, and open, and $\phi: D^{\mathbb{N}} \rightarrow Y$ is a continuous homomorphism from $\operatorname{Cnvg}_{X}\left(D^{\mathbb{N}}\right)$ to $\operatorname{Cnvg}(Y)$. Then there is a continuous extension of $\phi$ to $\operatorname{Ext}_{X}\left(D^{\mathbb{N}}\right)$.

Proof. Given a point $x \in X$, we say that a sequence $\left(X_{n}\right)_{n \in \mathbb{N}}$ of subsets of $X$ converges to $x$, or $X_{n} \rightarrow x$, if for every open neighborhood $U \subseteq X$ of $x$, there exists $n \in \mathbb{N}$ for which $\bigcup_{m \geq n} X_{m} \subseteq U$.

Lemma 3.5. Suppose that $t \in D^{<\mathbb{N}}$ and $x \in \sim D$. Then there exists $y_{t, x} \in Y$ such that $\mathbf{d}(n) \rightarrow x \Longrightarrow \phi\left(\mathcal{N}_{t \frown(\mathbf{d}(n))}\right) \rightarrow y_{t, x}$ for all $\mathbf{d} \in D^{\mathbb{N}}$.

Proof. Note that if $\mathbf{d} \in D^{\mathbb{N}}, \mathbf{d}(n) \rightarrow x$, and $y_{n} \in \phi\left(\mathcal{N}_{t \wedge(\mathbf{d}(n))}\right)$ for all $n \in \mathbb{N}$, then there exists $y_{t, x} \in Y$ for which $y_{n} \rightarrow y_{t, x}$. If there exists $\mathbf{c} \in D^{\mathbb{N}}$ such that $\mathbf{c}(n) \rightarrow x$ and $\phi\left(\mathcal{N}_{t \frown(\mathbf{c}(n))}\right) \not \rightarrow y_{t, x}$, then there exist an infinite set $N \subseteq \mathbb{N}$, an open neighborhood $V \subseteq Y$ of $y_{t, x}$, and points $y_{n} \in \phi\left(\mathcal{N}_{t \frown(\mathbf{c}(n))}\right) \backslash V$ for all $n \in N$. By thinning down $N$, we can assume that it is co-infinite. Set $\mathbf{b}=(\mathbf{c} \uparrow N) \cup(\mathbf{d} \uparrow \sim N)$, and observe that $\mathbf{b}(n) \rightarrow x$ but $(\phi(\mathbf{b}(n)))_{n \in \mathbb{N}}$ does not converge, a contradiction.

To see that the extension given by $\bar{\phi}(t \frown(x))=y_{t, x}$ is continuous, suppose that $\mathbf{d} \in \operatorname{Ext}_{X}\left(D^{\mathbb{N}}\right)$ and $V \subseteq Y$ is an open neighborhood of $\bar{\phi}(\mathbf{d})$, and fix an open neighborhood $W \subseteq Y$ of $\bar{\phi}(\mathbf{d})$ whose closure is 
contained in $V$. If $\mathbf{d} \in D^{\mathbb{N}}$, then the continuity of $\phi$ yields $n \in \mathbb{N}$ for which $\phi\left(\mathcal{N}_{\mathbf{d} \mid n}\right) \subseteq W$, in which case $\bar{\phi}\left(\mathcal{N}_{\mathbf{d} \mid n, X}\right) \subseteq \overline{\phi\left(\mathcal{N}_{\mathbf{d} \mid n}\right)} \subseteq \bar{W} \subseteq V$. Otherwise, there exists $t \in D^{<\mathbb{N}}$ and $x \in \sim D$ for which $\mathbf{d}=t \frown(x)$, so Lemma 3.5 yields an open neighborhood $U \subseteq X$ of $x$ for which $\phi\left(\mathcal{N}_{t, U} \cap D^{\mathbb{N}}\right) \subseteq W$, in which case $\bar{\phi}\left(\mathcal{N}_{t, U}\right) \subseteq \overline{\phi\left(\mathcal{N}_{t, U}\right)} \subseteq \bar{W} \subseteq V$.

\section{Countable unions of hyperrectangles}

A hyperrectangular homomorphism from a sequence $\left(R_{i}\right)_{i \in I}$ of subsets of $\prod_{d \in D} X_{d}$ to a sequence $\left(S_{i}\right)_{i \in I}$ of subsets of $\prod_{d \in D} Y_{d}$ is a function $\phi$ of the form $\prod_{d \in D} \phi_{d}$, where $\phi_{d}: \bigcup_{i \in I} \operatorname{proj}_{d}\left(R_{i}\right) \rightarrow Y_{d}$ for all $d \in D$, such that $\phi\left(R_{i}\right) \subseteq S_{i}$ for all $i \in I$.

We use $\mathbb{H}_{(D \times \mathbb{N})^{\mathbb{N}}, \infty}$ to denote the $D$-dimensional dihypergraph on $\operatorname{Ext}_{D \times \mathbb{N}_{*}}\left((D \times \mathbb{N})^{\mathbb{N}}\right)$ consisting of all sequences $(t \frown((d, \infty)))_{d \in D}$, where $t \in(D \times \mathbb{N})^{<\mathbb{N}}$.

Given a sequence $\left(X_{d}\right)_{d \in D}$ and disjoint sets $R, S \subseteq \prod_{d \in D} X_{d}$, let $H_{R, S}$ denote the $(D \times \mathbb{N})$-dimensional dihypergraph on $R$ consisting of all sequences $\left(\left(x_{c, d, n}\right)_{c \in D}\right)_{(d, n) \in D \times \mathbb{N}}$ of elements of $R$ with the property that $\bar{x}_{d}=\lim _{n \rightarrow \infty} x_{d, d, n}$ exists for all $d \in D$ and $\left(\bar{x}_{d}\right)_{d \in D} \in S$.

Proposition 4.1. Suppose that $D$ is a discrete set, $\left(X_{d}\right)_{d \in D}$ is a sequence of metric spaces, and $R, S \subseteq \prod_{d \in D} X_{d}$ are disjoint.

(1) The $(D \times \mathbb{N})$-dimensional dihypergraph $H_{R, S}$ is box open.

(2) There is an $\aleph_{0}$-coloring of $H_{R, S}$ if and only if there is a countable union of closed hyperrectangles separating $R$ from $S$.

(3) There is a continuous homomorphism from $\mathbb{H}_{(D \times \mathbb{N})^{\mathbb{N}}}$ to $H_{R, S}$ if and only if there is a continuous hyperrectangular homomorphism from $\left(\triangle^{D}\left((D \times \mathbb{N})^{\mathbb{N}}\right), \mathbb{H}_{(D \times \mathbb{N})^{\mathbb{N}}, \infty}\right)$ to $(R, S)$.

Proof. To see (1), note that if $\left(\left(x_{c, d, n}\right)_{c \in D}\right)_{(d, n) \in D \times \mathbb{N}} \in H_{R, S}, \epsilon_{n} \rightarrow 0$, and $U_{d, n}=\left\{\left(x_{c}\right)_{c \in D} \in R \mid \rho_{X_{d}}\left(x_{d}, x_{d, d, n}\right)<\epsilon_{n}\right\}$ for all $(d, n) \in D \times \mathbb{N}$, then $\prod_{(d, n) \in D \times \mathbb{N}} U_{d, n} \subseteq H_{R, S}$.

To see (2), note first that if $Q \subseteq R$ and $\left(\bar{x}_{d}\right)_{d \in D} \in \prod_{d \in D} \overline{\operatorname{proj}_{d}(Q)}$, then there are sequences $\left(x_{d, n}\right)_{n \in \mathbb{N}}$ of elements of $\operatorname{proj}_{d}(Q)$ such that $x_{d, n} \rightarrow \bar{x}_{d}$ for all $d \in D$, so there are sequences $\left(x_{c, d, n}\right)_{c \in D} \in Q$ such that $x_{d, d, n}=x_{d, n}$ for all $(d, n) \in D \times \mathbb{N}$, thus $x_{d, d, n} \rightarrow \bar{x}_{d}$ for all $d \in D$. It follows that if $Q$ is $H_{R, S}$-independent, then $\prod_{d \in D} \overline{\operatorname{proj}_{d}(Q)}$ and $S$ are disjoint, so if $c: X \rightarrow \mathbb{N}$ is an $\aleph_{0}$-coloring of $H_{R, S}$, then the union of the closed hyperrectangles $\prod_{d \in D} \overline{\operatorname{proj}_{d}\left(c^{-1}(\{n\})\right)}$ separates $R$ from $S$. Conversely, suppose that $F_{d}$ is a closed subset of $X_{d}$ for all $d \in D$ and $S \cap \prod_{d \in D} F_{d}=\emptyset$. If $\left(\left(x_{c, d, n}\right)_{c \in D}\right)_{(d, n) \in D \times \mathbb{N}}$ is a sequence of elements of $\prod_{c \in D} F_{c}$ such that $\bar{x}_{d}=\lim _{n \rightarrow \infty} x_{d, d, n}$ exists for all $d \in D$, 
then $\left(\bar{x}_{d}\right)_{d \in D} \in \prod_{d \in D} F_{d}$, so $\left(\bar{x}_{d}\right)_{d \in D} \notin S$, thus $R \cap \prod_{d \in D} F_{d}$ is $H_{R, S^{-}}$ independent. Hence if there is a countable union of closed hyperrectangles separating $R$ from $S$, then there is an $\aleph_{0}$-coloring of $H_{R, S}$.

To see (3), suppose first that $\prod_{d \in D} \phi_{d}$ is a continuous hyperrectangular homomorphism from $\left(\triangle^{D}\left((D \times \mathbb{N})^{\mathbb{N}}\right), \mathbb{H}_{(D \times \mathbb{N})^{\mathbb{N}}, \infty}\right)$ to $(R, S)$, and define $\phi:(D \times \mathbb{N})^{\mathbb{N}} \rightarrow \prod_{d \in D} X_{d}$ by $\phi(\mathbf{d})=\left(\phi_{d}(\mathbf{d})\right)_{d \in D}$. Clearly $\phi$ is continuous and $\phi\left((D \times \mathbb{N})^{\mathbb{N}}\right)=\left(\prod_{d \in D} \phi_{d}\right)\left(\triangle^{D}\left((D \times \mathbb{N})^{\mathbb{N}}\right)\right) \subseteq R$. To see that $\phi$ is a homomorphism from $\mathbb{H}_{(D \times \mathbb{N})^{\mathbb{N}}}$ to $H_{R, S}$, we need only note that $\left(\lim _{n \rightarrow \infty} \phi_{d}\left(\mathcal{N}_{t \frown((d, n))}\right)\right)_{d \in D}=\left(\phi_{d}(t \frown((d, \infty)))\right)_{d \in D}$ for all $t \in(D \times \mathbb{N})^{<\mathbb{N}}$, since $\left(\prod_{d \in D} \phi_{d}\right)\left(\mathbb{H}_{(D \times \mathbb{N})^{\mathbb{N}}, \infty}\right) \subseteq S$. Conversely, suppose that $\phi:(D \times \mathbb{N})^{\mathbb{N}} \rightarrow R$ is a continuous homomorphism from $\mathbb{H}_{(D \times \mathbb{N})^{\mathbb{N}}}$ to $H_{R, S}$. Then each of the functions $\operatorname{proj}_{d} \circ \phi$ is a continuous homomorphism from $\operatorname{Cnvg}_{(D \times \mathbb{N}) \cup\{(d, \infty)\}}\left((D \times \mathbb{N})^{\mathbb{N}}\right)$ to $\operatorname{Cnvg}\left(X_{d}\right)$, so Proposition 3.4 yields continuous extensions $\phi_{d}: \operatorname{Ext}_{(D \times \mathbb{N}) \cup\{(d, \infty)\}}\left((D \times \mathbb{N})^{\mathbb{N}}\right) \rightarrow X_{d}$ for all $d \in D$, and clearly $\prod_{d \in D} \phi_{d}$ is a hyperrectangular homomorphism from $\left(\triangle^{D}\left((D \times \mathbb{N})^{\mathbb{N}}\right), \mathbb{H}_{(D \times \mathbb{N})^{\mathbb{N}}, \infty}\right)$ to $(R, S)$.

In particular, we obtain the following.

Theorem $4.2\left(\mathrm{OGD}^{\mathbb{N}}(\Gamma)\right)$. Suppose that $D$ is a countable discrete set, $\left(X_{d}\right)_{d \in D}$ is a sequence of metric spaces, $R, S \subseteq \prod_{d \in D} X_{d}$ are disjoint, and $R \in \Gamma$. Then exactly one of the following holds:

(1) There is a countable union of closed hyperrectangles separating $R$ from $S$.

(2) There is a continuous hyperrectangular homomorphism from $\left(\triangle^{D}\left((D \times \mathbb{N})^{\mathbb{N}}\right), \mathbb{H}_{(D \times \mathbb{N})^{\mathbb{N}}, \infty}\right)$ to $(R, S)$.

Proof. This follows from Proposition 4.1.

The special cases of Theorem 4.2 where $D=1$ and $\Gamma$ is either the family of analytic spaces or the family of separable spaces easily yield the Kechris-Louveau-Woodin generalizations of Hurewicz's characterization of the circumstances under which two sets can be separated by an $F_{\sigma}$ set.

The special case of Theorem 4.2 where $D=2$ and $\Gamma$ is the family of analytic spaces easily yields the Lecomte-Zeleny characterization of the circumstances under which an analytic subset of the plane can be separated from another subset of the plane by a countable union of closed rectangles. The special case where $D=2$ and $\Gamma$ is the family of separable spaces easily yields the generalization in which analyticity is weakened to separability under the axiom of determinacy. 
Proposition 4.3. Suppose that $D$ is a discrete set of cardinality at least two, $X$ is a metric space, and $H$ is a D-dimensional dihypergraph on $X$.

(1) There is a countable union of closed hyperrectangles separating $\triangle^{D}(X)$ from $H$ if and only if there is a $\boldsymbol{\Delta}_{2}^{0}$-measurable $\aleph_{0}$ coloring of $H$.

(2) There is a continuous hyperrectangular homomorphism from $\left(\triangle^{D}\left((D \times \mathbb{N})^{\mathbb{N}}\right), \mathbb{H}_{(D \times \mathbb{N})^{\mathbb{N}}, \infty}\right)$ to $\left(\triangle^{D}(X), H\right)$ if and only if there is a continuous homomorphism from $\mathbb{H}_{(D \times \mathbb{N})^{\mathbb{N}}, \infty}$ to $H$.

Proof. To see (1), note first that if $\left(F_{d, n}\right)_{(d, n) \in D \times \mathbb{N}}$ is a sequence of closed subsets of $X$ for which $\bigcup_{n \in \mathbb{N}} \prod_{d \in D} F_{d, n}$ separates $\triangle^{D}(X)$ from $H$, then the $H$-independent closed sets $\bigcap_{d \in D} F_{d, n}$ cover $X$, so there is a $\boldsymbol{\Delta}_{2}^{0}$-measurable $\aleph_{0}$-coloring of $H$. Conversely, if there is a $\boldsymbol{\Delta}_{2}^{0}$ measurable $\aleph_{0}$-coloring of $H$, then there is a cover $\left(F_{n}\right)_{n \in \mathbb{N}}$ of $X$ by $H$-independent closed sets, in which case $\bigcup_{n \in \mathbb{N}} F_{n}^{D}$ is a countable union of closed hyperrectangles separating $\triangle^{D}(X)$ from $H$.

To see (2), note first that if $\phi: \operatorname{Ext}_{D \times \mathbb{N}_{*}}\left((D \times \mathbb{N})^{\mathbb{N}}\right) \rightarrow X$ is a homomorphism from $\mathbb{H}_{(D \times \mathbb{N})^{\mathbb{N}}, \infty}$ to $H$, and $\phi_{d}$ is the restriction of $\phi$ to $\operatorname{Ext}_{(D \times \mathbb{N}) \cup\{(d, \infty)\}}\left((D \times \mathbb{N})^{\mathbb{N}}\right)$ for all $d \in D$, then $\prod_{d \in D} \phi_{d}$ is a hyperrectangular homomorphism from $\left(\triangle^{D}\left((D \times \mathbb{N})^{\mathbb{N}}\right), \mathbb{H}_{(D \times \mathbb{N})^{\mathbb{N}}, \infty}\right)$ to $\left(\triangle^{D}(X), H\right)$. Conversely, observe that if $\prod_{d \in D} \phi_{d}$ is a continuous hyperrectangular homomorphism from $\left(\triangle^{D}\left((D \times \mathbb{N})^{\mathbb{N}}\right), \mathbb{H}_{(D \times \mathbb{N})^{\mathbb{N}}, \infty}\right)$ to $\left(\triangle^{D}(X), H\right)$, then the function $\phi=\bigcup_{d \in D} \phi_{d}$ is a continuous homomorphism from $\mathbb{H}_{(D \times \mathbb{N})^{\mathbb{N}}, \infty}$ to $H$.

In particular, we obtain the following.

Theorem $4.4\left(\mathrm{OGD}^{\mathbb{N}}(\Gamma)\right)$. Suppose that $D$ is a countable discrete set of cardinality at least two, $X$ is a metric space in $\Gamma$, and $H$ is a $D$ dimensional dihypergraph on $X$. Then exactly one of the following holds:

(1) There is a $\boldsymbol{\Delta}_{2}^{0}$-measurable $\aleph_{0}$-coloring of $H$.

(2) There is a continuous homomorphism from $\mathbb{H}_{(D \times \mathbb{N})^{\mathbb{N}}, \infty}$ to $H$.

Proof. This follows from Propositions 4.1 and 4.3.

The special case of Theorem 4.4 where $D=2$ and $\Gamma$ is the family of analytic spaces easily yields the Lecomte-Zeleny characterization of the circumstances under which a graph on an analytic metric space has a $\boldsymbol{\Delta}_{2}^{0}$-measurable $\aleph_{0}$-coloring. The special case where $D=2$ and $\Gamma$ is the family of separable spaces easily yields the generalization in which analyticity is weakened to separability under the axiom of determinacy. 


\section{Sigma-COntinuous Functions With ClOSED Witnesses}

A reduction of a set $R \subseteq X^{D}$ to a set $S \subseteq Y^{D}$ is a single function $\phi: X \rightarrow Y$ that is a homomorphism from $R$ to $S$ and from $\sim R$ to $\sim S$.

Given a function $\pi: X \rightarrow Y$, let $H_{\pi}$ denote the $\mathbb{N}$-dimensional dihypergraph on $\operatorname{graph}(\pi)$ consisting of all sequences $\left(\left(x_{n}, y_{n}\right)\right)_{n \in \mathbb{N}}$ of elements of $\operatorname{graph}(\pi)$ with the property that $\bar{x}=\lim _{n \rightarrow \infty} x_{n}$ exists but $\pi(\bar{x}) \notin \overline{\left\{y_{n} \mid n \in \mathbb{N}\right\}}$.

Proposition 5.1. Suppose that $X$ and $Y$ are metric spaces and $\pi: X \rightarrow Y$.

(1) The dihypergraph $H_{\pi}$ is box open.

(2) There is an $\aleph_{0}$-coloring of $H_{\pi}$ if and only if $\pi$ is $\sigma$-continuous with closed witnesses.

(3) There is a continuous homomorphism from $\mathbb{H}_{\mathbb{N}^{\mathbb{N}}}$ to $H_{\pi}$ if and only if there is a continuous function $\psi: \operatorname{Ext}_{\mathbb{N}_{*}}\left(\mathbb{N}^{\mathbb{N}}\right) \rightarrow X$ with the property that $\pi \circ \psi$ is a reduction of $\mathbb{N}^{\mathbb{N}}$ to a closed set and $(\pi \circ \psi) \uparrow \mathbb{N}^{\mathbb{N}}$ is continuous.

Proof. To see (1), note that if $\left(\left(x_{n}, y_{n}\right)\right)_{n \in \mathbb{N}} \in H_{\pi}$ and $\bar{x}=\lim _{n \rightarrow \infty} x_{n}$, then there exists $\epsilon>0$ such that $\rho_{Y}\left(\pi(\bar{x}), y_{n}\right) \geq 2 \epsilon$ for all $n \in \mathbb{N}$. Fix positive real numbers $\epsilon_{n} \rightarrow 0$, and observe that the intersection of $\operatorname{graph}(\pi)$ with $\prod_{n \in \mathbb{N}} \mathcal{B}_{X}\left(x_{n}, \epsilon_{n}\right) \times \mathcal{B}_{Y}\left(y_{n}, \epsilon\right)$ is contained in $H_{\pi}$.

To see (2), note first that if $F \subseteq X$ is a closed set on which $\pi$ is continuous, then $\operatorname{graph}(\pi) \cap(F \times Y)$ is $H_{\pi}$-independent, so if $\pi$ is $\sigma$-continuous with closed witnesses, then there is a Borel $\aleph_{0}$-coloring of $H_{\pi}$. Conversely, if $R \subseteq \operatorname{graph}(\pi)$ and $\left(\bar{x}_{n}\right)_{n \in \mathbb{N}}$ is a convergent sequence of elements of the closure of $\operatorname{proj}_{X}(R)$, then there are sequences $\left(x_{m, n}\right)_{m \in \mathbb{N}}$ of elements of $\operatorname{proj}_{X}(R)$ such that $x_{m, n} \rightarrow \bar{x}_{n}$ for all $n \in \mathbb{N}$. If $R$ is $H_{\pi}$-independent, then $\pi\left(x_{m, n}\right) \rightarrow \pi\left(\bar{x}_{n}\right)$ for all $n \in \mathbb{N}$, so there is a function $f: \mathbb{N} \rightarrow \mathbb{N}$ such that $\rho_{X}\left(x_{f(n), n}, \bar{x}_{n}\right) \rightarrow 0$ and $\rho_{Y}\left(\pi\left(x_{f(n), n}\right), \pi\left(\bar{x}_{n}\right)\right) \rightarrow 0$. It follows that $\left(x_{f(n), n}\right)_{n \in \mathbb{N}}$ converges, so the fact that $R$ is $H_{\pi}$-independent ensures that $\pi\left(\lim _{n \rightarrow \infty} x_{f(n), n}\right)=$ $\lim _{n \rightarrow \infty} \pi\left(x_{f(n), n}\right)$, in which case $\pi\left(\lim _{n \rightarrow \infty} \bar{x}_{n}\right)=\lim _{n \rightarrow \infty} \pi\left(\bar{x}_{n}\right)$, thus $\pi \uparrow \overline{\operatorname{proj}_{X}(R)}$ is continuous. In particular, it follows that if there is an $\aleph_{0}$-coloring of $H_{\pi}$, then $\pi$ is $\sigma$-continuous with closed witnesses.

To see (3), note first that if $\psi: \operatorname{Ext}_{\mathbb{N}_{*}}\left(\mathbb{N}^{\mathbb{N}}\right) \rightarrow X$ is continuous, $\pi \circ \psi$ is a reduction of $\mathbb{N}^{\mathbb{N}}$ to a closed set, $(\pi \circ \psi) \uparrow \mathbb{N}^{\mathbb{N}}$ is continuous, and $\left(\mathbf{d}_{n}\right)_{n \in \mathbb{N}} \in \mathbb{H}_{\mathbb{N}^{\mathbb{N}}}$, then $(\pi \circ \psi)\left(\lim _{n \rightarrow \infty} \mathbf{d}_{n}\right) \notin \overline{\left\{(\pi \circ \psi)\left(\mathbf{d}_{n}\right) \mid n \in \mathbb{N}\right\}}$, so the continuity of $\psi$ ensures that $\left(\left(\psi\left(\mathbf{d}_{n}\right),(\pi \circ \psi)\left(\mathbf{d}_{n}\right)\right)\right)_{n \in \mathbb{N}} \in H_{\pi}$, thus $\left(\psi \uparrow \mathbb{N}^{\mathbb{N}}\right) \times\left((\pi \circ \psi) \uparrow \mathbb{N}^{\mathbb{N}}\right)$ is a homomorphism from $\mathbb{H}_{\mathbb{N}^{\mathbb{N}}}$ to $H_{\pi}$.

Conversely, suppose that $\phi: \mathbb{N}^{\mathbb{N}} \rightarrow \operatorname{graph}(\pi)$ is a continuous homomorphism from $\mathbb{H}_{\mathbb{N}^{\mathbb{N}}}$ to $H_{\pi}$, and set $\phi_{X}=\operatorname{proj}_{X} \circ \phi$ and $\phi_{Y}=\operatorname{proj}_{Y} \circ \phi$. As the definition of $H_{\pi}$ ensures that $\phi_{X}$ is a homomorphism from 
$\operatorname{Cnvg}_{\mathbb{N}_{*}}\left(\mathbb{N}^{\mathbb{N}}\right)$ to $\operatorname{Cnvg}(X)$, Proposition 3.4 yields a continuous exten$\operatorname{sion} \bar{\phi}_{X}: \operatorname{Ext}_{\mathbb{N}_{*}}\left(\mathbb{N}^{\mathbb{N}}\right) \rightarrow X$.

If there exists $t \in \mathbb{N}^{<\mathbb{N}}$ for which $\phi_{Y} \uparrow \mathcal{N}_{t}$ is constant, then the function $\psi: \operatorname{Ext}_{\mathbb{N}_{*}}\left(\mathbb{N}^{\mathbb{N}}\right) \rightarrow X$ given by $\psi(\mathbf{d})=\bar{\phi}_{X}(t \frown \mathbf{d})$ is continuous and $\pi \circ \psi$ is a reduction of $\mathbb{N}^{\mathbb{N}}$ to a singleton, so we can assume that

$$
\forall t \in \mathbb{N}^{<\mathbb{N}} \phi_{Y}\left(\mathcal{N}_{t}\right) \text { is infinite. }
$$

If there exists $t \in \mathbb{N}^{<\mathbb{N}}$ for which $\left(\pi \circ \bar{\phi}_{X}\right)\left(\mathcal{N}_{t, \mathbb{N}_{*}} \backslash \mathbb{N}^{\mathbb{N}}\right)$ is finite, then by appealing to $(\dagger)$ and extending $t$, we can ensure that $\overline{\phi_{Y}\left(\mathcal{N}_{t}\right)}$ and $\left(\pi \circ \bar{\phi}_{X}\right)\left(\mathcal{N}_{t, \mathbb{N}_{*}} \backslash \mathbb{N}^{\mathbb{N}}\right)$ are disjoint, so the function $\psi: \operatorname{Ext}_{\mathbb{N}_{*}}\left(\mathbb{N}^{\mathbb{N}}\right) \rightarrow X$ given by $\psi(\mathbf{d})=\bar{\phi}_{X}(t \frown \mathbf{d})$ is continuous, $\pi \circ \psi$ is a reduction of $\mathbb{N}^{\mathbb{N}}$ to $\overline{\phi_{Y}\left(\mathcal{N}_{t}\right)}$, and $(\pi \circ \psi)(\mathbf{d})=\phi_{Y}(t \frown \mathbf{d})$ for all $\mathbf{d} \in \mathbb{N}^{\mathbb{N}}$. We can therefore additionally assume that

$$
\forall t \in \mathbb{N}^{<\mathbb{N}}\left(\pi \circ \bar{\phi}_{X}\right)\left(\mathcal{N}_{t, \mathbb{N}_{*}} \backslash \mathbb{N}^{\mathbb{N}}\right) \text { is infinite. }
$$

Fix positive real numbers $\epsilon_{n} \rightarrow 0$, as well as an enumeration $\left(t_{k}\right)_{k \in \mathbb{N}}$ of $\mathbb{N}^{<\mathbb{N}}$ such that $t_{j} \sqsubseteq t_{k} \Longrightarrow j \leq k$ for all $j, k \in \mathbb{N}$. We say that a sequence $\left(X_{n}\right)_{n \in \mathbb{N}}$ of subsets of $X$ is closed and discrete if there are no convergent sequences in $\prod_{n \in \mathbb{N}} X_{n}$. We will recursively construct functions $f: \mathbb{N}^{<\mathbb{N}} \rightarrow \mathbb{N}^{<\mathbb{N}}$ and $g: \mathbb{N}^{<\mathbb{N}} \backslash\{\emptyset\} \rightarrow \mathbb{N}^{<\mathbb{N}}$ such that, for all $k \in \mathbb{N}$, the following conditions hold:

(a) $\forall n \in \mathbb{N} f\left(t_{k}\right) \sqsubset g\left(t_{k} \frown(n)\right)$.

(b) $\forall m \neq n g\left(t_{k} \frown(m)\right)\left(\left|f\left(t_{k}\right)\right|\right) \neq g\left(t_{k} \frown(n)\right)\left(\left|f\left(t_{k}\right)\right|\right)$.

(c) $\left(\phi_{Y}\left(\mathcal{N}_{g\left(t_{k} \wedge(n)\right)}\right)\right)_{n \in \mathbb{N}}$ is either closed-and-discrete or convergent.

(d) $g\left(t_{k+1}\right) \sqsubseteq f\left(t_{k+1}\right)$.

(e) $\forall j \leq k\left(\pi \circ \bar{\phi}_{X}\right)\left(f\left(t_{j}\right) \frown(\infty)\right) \notin \overline{\phi_{Y}\left(\mathcal{N}_{f\left(t_{k+1}\right)}\right)}$.

(f) $\forall j \leq k\left(\pi \circ \bar{\phi}_{X}\right)\left(f\left(t_{k+1}\right) \frown(\infty)\right) \neq \lim _{n \rightarrow \infty} \phi_{Y}\left(\mathcal{N}_{g\left(t_{j} \wedge(n)\right)}\right)$.

We begin by setting $f(\emptyset)=\emptyset$.

Suppose now that $k \in \mathbb{N}$ and we have already found $f\left(t_{k}\right)$, as well as $g\left(t_{j} \frown(n)\right)$ for all $j<k$ and $n \in \mathbb{N}$, and fix extensions $\mathbf{d}_{k, n} \in \mathbb{N}^{\mathbb{N}}$ of $f\left(t_{k}\right) \frown(n)$ for all $n \in \mathbb{N}$. Then there is an injective sequence $\left(i_{k, n}\right)_{n \in \mathbb{N}}$ of natural numbers such that $\left(\left\{\phi_{Y}\left(\mathbf{d}_{k, i_{k, n}}\right)\right\}\right)_{n \in \mathbb{N}}$ is either closed-and-discrete or convergent, and continuity yields extensions $g\left(t_{k} \frown(n)\right) \sqsubset \mathbf{d}_{k, i_{k, n}}$ of $f\left(t_{k}\right) \frown\left(i_{k, n}\right)$ with the property that $\phi_{Y}\left(\mathcal{N}_{g\left(t_{k} \wedge(n)\right)}\right) \subseteq \mathcal{B}_{Y}\left(\phi_{Y}\left(\mathbf{d}_{k, i_{k, n}}\right), \epsilon_{n}\right)$ for all $n \in \mathbb{N}$. By $(\dagger)$, there is an extension $\mathbf{d}_{k} \in \mathbb{N}^{\mathbb{N}}$ of $g\left(t_{k+1}\right)$ such that $\phi_{Y}\left(\mathbf{d}_{k}\right) \neq\left(\pi \circ \bar{\phi}_{X}\right)\left(f\left(t_{j}\right) \frown(\infty)\right)$ for all $j \leq k$, and therefore an extension $u_{k} \sqsubset \mathbf{d}_{k}$ of $g\left(t_{k+1}\right)$ such that $\left(\pi \circ \bar{\phi}_{X}\right)\left(f\left(t_{j}\right) \frown(\infty)\right) \notin \overline{\phi_{Y}\left(\mathcal{N}_{u_{k}}\right)}$ for all $j \leq k$. By $(\ddagger)$, there is an extension $f\left(t_{k+1}\right)$ of $u_{k}$ satisfying condition (f), thereby completing the recursive construction. 
Conditions (a), (b), and (d) ensure that the function $\phi_{X} \circ \phi^{\prime}$ is a homomorphism from $\operatorname{Cnvg}_{\mathbb{N}_{*}}\left(\mathbb{N}^{\mathbb{N}}\right)$ to $\operatorname{Cnvg}(X)$, where $\phi^{\prime}: \mathbb{N}^{\mathbb{N}} \rightarrow \mathbb{N}^{\mathbb{N}}$ is given by $\phi^{\prime}(\mathbf{d})=\bigcup_{n \in \mathbb{N}} f(\mathbf{d}\lceil n)$. Proposition 3.4 therefore yields a continuous extension $\psi: \operatorname{Ext}_{\mathbb{N}_{*}}\left(\mathbb{N}^{\mathbb{N}}\right) \rightarrow X$. As $(\pi \circ \psi) \uparrow \mathbb{N}^{\mathbb{N}}=\phi_{Y} \circ \phi^{\prime}$, it only remains to show that $\pi \circ \psi$ is a reduction of $\mathbb{N}^{\mathbb{N}}$ to a closed set.

Suppose, towards a contradiction, that there exists $k \in \mathbb{N}$ with the property that $(\pi \circ \psi)\left(t_{k} \frown(\infty)\right) \in \overline{(\pi \circ \psi)\left(\mathbb{N}^{\mathbb{N}}\right)}$. As conditions (a), (b), and $(d)$ ensure that

$$
\begin{aligned}
(\pi \circ \psi)\left(t_{k} \frown(\infty)\right) & =\pi\left(\lim _{n \rightarrow \infty}\left(\phi_{X} \circ \phi^{\prime}\right)\left(\mathcal{N}_{t_{k} \frown(n)}\right)\right) \\
& =\pi\left(\lim _{n \rightarrow \infty} \phi_{X}\left(\mathcal{N}_{f\left(t_{k} \frown(n)\right)}\right)\right) \\
& =\left(\pi \circ \bar{\phi}_{X}\right)\left(f\left(t_{k}\right) \frown(\infty)\right),
\end{aligned}
$$

it follows that $\left(\pi \circ \bar{\phi}_{X}\right)\left(f\left(t_{k}\right) \frown(\infty)\right) \in \overline{\phi_{Y}\left(\mathbb{N}^{\mathbb{N}}\right)}$. As condition (e) ensures that $\left(\pi \circ \bar{\phi}_{X}\right)\left(f\left(t_{k}\right) \frown(\infty)\right) \notin \overline{\phi_{Y}\left(\mathcal{N}_{f\left(t_{j}\right)}\right)}$ for all $j>k$, and condition (f) implies that if $i<k$ and $\left(\pi \circ \bar{\phi}_{X}\right)\left(f\left(t_{k}\right) \frown(\infty)\right) \in \overline{\phi_{Y}\left(\mathcal{N}_{f\left(t_{i}\right)}\right)}$ then there exists $j>i$ for which $\left(\pi \circ \bar{\phi}_{X}\right)\left(f\left(t_{k}\right) \frown(\infty)\right) \in \overline{\phi_{Y}\left(\mathcal{N}_{f\left(t_{j}\right)}\right)}$, it follows that $\left(\pi \circ \bar{\phi}_{X}\right)\left(f\left(t_{k}\right) \frown(\infty)\right) \in \overline{\phi_{Y}\left(\mathcal{N}_{f\left(t_{k}\right)}\right)} \backslash \bigcup_{n \in \mathbb{N}} \overline{\phi_{Y}\left(\mathcal{N}_{f\left(t_{k} \frown(n)\right)}\right)}$. Conditions (a), (b), and (d) therefore yield extensions $\mathbf{c}_{n} \in \mathbb{N}^{\mathbb{N}}$ of $f\left(t_{k}\right) \frown(n)$ such that $\pi\left(\lim _{n \rightarrow \infty} \phi_{X}\left(\mathbf{c}_{n}\right)\right) \in \overline{\left\{\left(\pi \circ \phi_{X}\right)\left(\mathbf{c}_{n}\right)\right\}}$, contradicting the fact that $\phi$ is a homomorphism from $\mathbb{H}_{\mathbb{N}^{\mathbb{N}}}$ to $H_{\pi}$.

In particular, we obtain the following.

Theorem $5.2\left(\mathrm{OGD}^{\mathbb{N}}(\Gamma)\right)$. Suppose that $X$ and $Y$ are separable metric spaces, $X \in \Gamma$, and $\pi: X \rightarrow Y$ is Borel. Then exactly one of the following holds:

(1) The function $\pi$ is $\sigma$-continuous with closed witnesses.

(2) There is a continuous function $\psi: \operatorname{Ext}_{\mathbb{N}_{*}}\left(\mathbb{N}^{\mathbb{N}}\right) \rightarrow X$ with the property that $\pi \circ \psi$ is a reduction of $\mathbb{N}^{\mathbb{N}}$ to a closed set and $(\pi \circ \psi) \uparrow \mathbb{N}^{\mathbb{N}}$ is continuous.

Proof. This follows from Proposition 5.1.

The special case of Theorem 5.2 where $\Gamma$ is the family of analytic spaces easily yields the Jayne-Rogers characterization of the circumstances under which a function from an analytic metric space to a separable metric space is $\sigma$-continuous with closed witnesses. The special case where $\Gamma$ is the family of separable spaces easily yields the generalization in which analyticity is weakened to separability under the axiom of determinacy. 


\section{Cardinal invariants And Chromatic nUmbers}

We begin this section by noting a straightforward restriction on chromatic numbers of box-open hereditary $\mathbb{N}$-dimensional dihypergraphs.

Proposition 6.1. The chromatic number of $\mathbb{H}_{\mathbb{N}^{\mathbb{N}}}^{\prime}$ is $\mathfrak{d}$.

Proof. This follows from the fact that a subset of $\mathbb{N}^{\mathbb{N}}$ is $\mathbb{H}_{\mathbb{N}^{\mathbb{N}}}^{\prime}$-independent if and only if its closure is compact.

Proposition $6.2\left(\mathrm{OGD}^{\mathbb{N}}(\Gamma)\right)$. Suppose that $X$ is a Hausdorff space in $\Gamma$ and $H$ is a box-open hereditary $\mathbb{N}$-dimensional dihypergraph on $X$. Then either $\chi(H) \leq \aleph_{0}$ or $\chi(H) \geq \mathfrak{d}$.

Proof. This follows from Proposition 6.1 and the fact that every homomorphism from $\mathbb{H}_{\mathbb{N}^{\mathbb{N}}}$ to $H$ is a homomorphism from $\mathbb{H}_{\mathbb{N}^{\mathbb{N}}}^{\prime}$ to $H$.

As every analytic subset of a topological space is the union of $\mathfrak{d}$-many compact sets, the special case of Proposition 6.2 where $\Gamma$ is the family of analytic sets and $H$ is of the form $H_{X}$ ensures that if an analytic subset of a metric space is not contained in a $K_{\sigma}$ set, then $\mathfrak{d}$ is the least cardinal $\kappa$ for which it is contained in a union of $\kappa$-many compact sets.

As every analytic subset of a topological space is the union of $\mathfrak{d}$-many closed sets, the special case of Proposition 6.2 where $\Gamma$ is the family of analytic sets, $H$ is of the form $H_{R, S}$, and $D=1$ ensures that if an analytic subset of a metric space cannot be separated from another set by an $F_{\sigma}$ set, then $\mathfrak{d}$ is the least cardinal $\kappa$ for which it can be separated from the other set by a union of $\kappa$-many closed sets.

As every analytic set that is the graph of a function is the union of $\mathfrak{d}$-many compact sets that are graphs of functions, the special case of Proposition 6.2 where $\Gamma$ is the family of analytic sets and $H$ is of the form $H_{\pi}$ ensures that if a Borel function $\pi$ from an analytic metric space to a separable metric space is not $\sigma$-continuous with closed witnesses, then $\mathfrak{d}$ is the least cardinal $\kappa$ for which $X$ is the union of $\kappa$-many closed sets on which $\pi$ is continuous.

The special case of the last fact for Baire-class-one functions is due to Solecki. He established this by noting that the two elements of his basis for Baire-class-one functions that are not $\sigma$-continuous have the desired property. The above argument shows that this more sophisticated basis theorem is unnecessary to obtain the desired result; one need only observe that $\sigma$-continuity with closed witnesses can be characterized using a box-open hereditary $\mathbb{N}$-dimensional dihypergraph.

We next turn our attention to the computation of the chromatic number of $\mathbb{H}_{\mathbb{N}^{\mathbb{N}}}$ itself. For each partial function $f: D^{<\mathbb{N}} \rightarrow D$, define $\mathbf{D}_{f}=\left\{\mathbf{d} \in D^{\mathbb{N}} \mid \forall n \in \mathbb{N}(\mathbf{d}\lceil n \in \operatorname{dom}(f) \Longrightarrow \mathbf{d}(n) \neq f(\mathbf{d}\lceil n))\}\right.$. 
Proposition 6.3. Suppose that $D$ is a set of cardinality at least two. Then $\chi\left(\mathbb{H}_{D^{\mathbb{N}}}\right)=\min \left\{|\mathcal{F}| \mid \mathcal{F} \subseteq D^{D^{<\mathbb{N}}}\right.$ and $\left.D^{\mathbb{N}}=\bigcup_{f \in \mathcal{F}} \mathbf{D}_{f}\right\}$.

Proof. This follows from the fact that a set $X \subseteq D^{\mathbb{N}}$ is $\mathbb{H}_{D^{\mathbb{N}} \text {-independent }}$ if and only if there is a function $f: D^{<\mathbb{N}} \rightarrow D$ for which $X \subseteq \mathbf{D}_{f}$. $\otimes$

We now establish an analog of Proposition 6.2 without the assumption that $H$ is hereditary.

Proposition $6.4\left(\mathrm{OGD}^{D}(\Gamma)\right)$. Suppose that $D$ is a countable set of cardinality at least two, $X$ is a Hausdorff space in $\Gamma$, and $H$ is a boxopen D-dimensional dihypergraph on $X$. Then either $\chi(H) \leq \aleph_{0}$ or $\chi(H) \geq \operatorname{cov}(\mathcal{M})$.

Proof. It is sufficient to show that $\chi\left(\mathbb{H}_{D^{\mathbb{N}}}\right) \geq \operatorname{cov}(\mathcal{M})$, which follows from Proposition 6.3 and the observation that if $f: D^{<\mathbb{N}} \rightarrow D$, then $\mathbf{D}_{f}$ is meager with respect to the usual topology on $D^{\mathbb{N}}$.

Alternatively, one can obtain the above result by noting that if $H$ is a box-open $D$-dimensional dihypergraph on a Hausdorff space, $\mathcal{I}_{H}$ is the $\sigma$-ideal generated by the family of closed $H$-independent sets, and $\chi(H)>\aleph_{0}$, then $\chi(H)=\operatorname{cov}\left(\mathcal{I}_{H}\right)$.

Conversely, given a $\sigma$-ideal $\mathcal{I}$ on a topological space $X$, let $H_{\mathcal{I}}$ be the $\mathbb{N}$-dimensional dihypergraph on $X$ consisting of all sequences $\left(x_{n}\right)_{n \in \mathbb{N}}$ of elements of $X$ for which $\overline{\left\{x_{n} \mid n \in \mathbb{N}\right\}} \notin \mathcal{I}$.

Proposition 6.5. Suppose that $X$ is a separable metric space and $\mathcal{I}$ is a $\sigma$-ideal on $X$ generated by a family of closed subsets of $X$.

(1) If $\mathcal{I}$ covers $X$, then $H_{\mathcal{I}}$ is box open.

(2) If $\operatorname{cov}(\mathcal{I})>\aleph_{0}$, then $\chi\left(H_{\mathcal{I}}\right)=\operatorname{cov}(\mathcal{I})$.

Proof. To see (1), suppose that $\left(x_{n}\right)_{n \in \mathbb{N}} \in H_{\mathcal{I}}$, fix positive real numbers $\epsilon_{n} \rightarrow 0$, and observe that if $\left(y_{n}\right)_{n \in \mathbb{N}} \in \prod_{n \in \mathbb{N}} \mathcal{B}_{X}\left(x_{n}, \epsilon_{n}\right)$, then $\overline{\left\{x_{n} \mid n \in \mathbb{N}\right\}} \subseteq\left\{x_{n} \mid n \in \mathbb{N}\right\} \cup \overline{\left\{y_{n} \mid n \in \mathbb{N}\right\}}$, thus $\left(y_{n}\right)_{n \in \mathbb{N}} \in H_{\mathcal{I}}$.

To see $(2)$, note first that every $H_{\mathcal{I}}$-independent set is in $\mathcal{I}$, so $\operatorname{cov}(\mathcal{I}) \leq \chi\left(H_{\mathcal{I}}\right)$. Conversely, as every set in $\mathcal{I}$ is contained in the union of countably-many closed sets in $\mathcal{I}$, the fact that every closed set in $\mathcal{I}$ is $H_{\mathcal{I}}$-independent ensures that $\chi\left(H_{\mathcal{I}}\right) \leq \operatorname{cov}(\mathcal{I}) \cdot \aleph_{0}=\operatorname{cov}(\mathcal{I}) . \quad \otimes$

As a corollary, we also obtain an analog of Proposition 6.1 without the assumption that $H$ is hereditary.

Proposition 6.6. The chromatic number of $\mathbb{H}_{\mathbb{N}^{\mathbb{N}}}$ is $\operatorname{cov}(\mathcal{M})$.

Proof. Proposition 6.4 ensures that $\chi\left(\mathbb{H}_{\mathbb{N}^{\mathbb{N}}}\right) \geq \operatorname{cov}(\mathcal{M})$. As the open dihypergraph dichotomy yields a homomorphism from $\mathbb{H}_{\mathbb{N}^{\mathbb{N}}}$ to $H_{\mathcal{M}}$, Proposition 6.5 implies that $\chi\left(\mathbb{H}_{\mathbb{N}^{\mathbb{N}}}\right) \leq \operatorname{cov}(\mathcal{M})$. 
Stronger bounds can be obtained when $D$ is finite. In the special case that $D=2$, this is trivial.

Proposition $6.7(\mathrm{OGD}(\Gamma))$. Suppose that $X$ is a Hausdorff space in $\Gamma$ and $G$ is an open graph on $X$. Then either $\chi(G) \leq \aleph_{0}$ or $\chi(G)=\mathfrak{c}$.

Proof. It is sufficient to show that $\chi\left(\mathbb{H}_{2^{\mathbb{N}}}\right)=\mathfrak{c}$, which follows from the fact that $\mathbb{H}_{2^{\mathbb{N}}}=\mathbb{K}_{2^{\mathbb{N}}}$.

The case that $2<|D|<\aleph_{0}$ is substantially subtler, and related to the adaptive global prediction numbers considered in [Bla10, §10]. Our first observation is a straightforward analog of Proposition 6.4.

Proposition $6.8\left(\mathrm{OGD}^{D}(\Gamma)\right)$. Suppose that $D$ is a finite set of cardinality at least two, $X$ is a Hausdorff space in $\Gamma$, and $H$ is an open $D$-dimensional dihypergraph on $X$. Then either $\chi(H) \leq \aleph_{0}$ or $\chi(H) \geq$ $\operatorname{cov}(\mathcal{N})$.

Proof. It is sufficient to show that $\chi\left(\mathbb{H}_{D^{\mathbb{N}}}\right) \geq \operatorname{cov}(\mathcal{N})$, which follows from Proposition 6.3 and the observation that if $f: D^{<\mathbb{N}} \rightarrow D$ and $\mu$ is a strictly positive probability measure on $D$, then $\mathbf{D}_{f}$ is $\mu^{\mathbb{N}}$-null. $\otimes$

We next establish an analog of the main result of [Bre03]. We say that a partial function $u: \mathbb{N} \rightarrow D$ is a $D$-Silver condition if its domain is co-infinite, and we say that a set $X \subseteq D^{\mathbb{N}}$ is $D$-Silver null if every $D$ Silver condition $u: \mathbb{N} \rightarrow D$ extends to a $D$-Silver condition $v: \mathbb{N} \rightarrow D$ for which $\mathcal{N}_{v}$ is disjoint from $X$.

Theorem 6.9. Suppose that $D$ is a finite set of cardinality at least two, $X \subseteq D^{\mathbb{N}}$, and $\chi\left(\mathbb{H}_{D^{\mathbb{N}}}\lceil X)<\mathfrak{b}\right.$. Then $X$ is D-Silver null.

Proof. By Proposition 6.3, it is sufficient to show that if $\mathcal{F} \subseteq D^{D^{<\mathbb{N}}}$ has cardinality strictly less than $\mathfrak{b}$, then $\bigcup_{f \in \mathcal{F}} \mathbf{D}_{f}$ is $D$-Silver null. By a straightforward recursive construction of length $|D|$, we need only show that for all $d \in D$, every $D$-Silver condition $u: \mathbb{N} \rightarrow D$ extends to a $D$-Silver condition $v: \mathbb{N} \rightarrow D$ with the property that

$$
\forall f \in \mathcal{F} \forall \mathbf{d} \in \mathbf{D}_{f} \cap \mathcal{N}_{v} \forall^{\infty} n \in \sim \operatorname{dom}(v) d \neq f(\mathbf{d}\lceil n) .
$$

It is clearly sufficient to handle the special case that $u=\emptyset$.

For all $f \in \mathcal{F}$, fix a function $g_{f}: \mathbb{N} \rightarrow \mathbb{N}$ such that for all $n \in \mathbb{N}$ and $t \in D^{n}$, if there exists $i \in \mathbb{N}$ for which $d=f\left(t \frown(d)^{i}\right)$, then there is such an $i<g_{f}(n)$. Fix a function $g: \mathbb{N} \rightarrow \mathbb{N}$ eventually dominating $g_{f}$ for all $f \in \mathcal{F}$, set $h^{\prime}(0)=0$, and recursively define $h(n)=g\left(h^{\prime}(n)+1\right)$ and $h^{\prime}(n+1)=h^{\prime}(n)+1+h(n)$ for all $n \in \mathbb{N}$. Let $v$ be the function on $\sim\left\{h^{\prime}(n+1) \mid n \in \mathbb{N}\right\}$ with constant value $d$. 
To see that $v$ is as desired, note first that if $\mathbf{d} \in \mathcal{N}_{v}$, then a straightforward inductive argument reveals that $\mathbf{d}\left\lceil h^{\prime}(n+1)\right.$ is the concatenation of $\mathbf{d}\left\lceil\left(h^{\prime}(n)+1\right)\right.$ and $(d)^{h(n)}$, for all $n \in \mathbb{N}$. It follows that if $f \in \mathcal{F}$, $\mathbf{d} \in \mathbf{D}_{f}$, and $n \in \mathbb{N}$ is sufficiently large that $g_{f}\left(h^{\prime}(n)+1\right) \leq g\left(h^{\prime}(n)+1\right)$, then $d \neq f\left(\mathbf{d} \uparrow h^{\prime}(n+1)\right)$.

Theorem $6.10\left(\mathrm{OGD}^{D}(\Gamma)\right)$. Suppose that $D$ is a finite set of cardinality at least two, $X$ is a Hausdorff space in $\Gamma$, and $H$ is an open $D$ dimensional dihypergraph on $X$. Then either $\chi(H) \leq \aleph_{0}$ or $\chi(H) \geq \mathfrak{b}$.

Proof. It is sufficient to show that $\chi\left(\mathbb{H}_{D^{\mathbb{N}}}\right) \geq \mathfrak{b}$, which follows from Theorem 6.9.

We now establish an analog of the main result of [Kam00].

Theorem 6.11. It is consistent that $\chi\left(\mathbb{H}_{D^{\mathbb{N}}}\right)>\operatorname{cof}(\mathcal{N})$ for every finite set $D$ of cardinality at least two.

Proof. Given a natural number $D \geq 2$, we say that a subtree $T$ of $D^{<\mathbb{N}}$ is $D$-perfect if every element of $T$ has an extension $t \in T$ with the property that $\forall d \in D t \frown(d) \in T$. Let $S_{D}$ denote the set of such trees. Let $\leq$ denote the partial order on $S_{D}$ with respect to which $S \leq T$ if and only if $S \subseteq T$, and define $\mathbb{S}_{D}=\left(S_{D}, \leq\right)$. When $D=2$, this is just Sacks forcing, and the usual proof that the latter is proper and has the Sacks property works just as well for every $\mathbb{S}_{D}$.

Lemma 6.12. Suppose that $D \geq 2$ is a natural number, $G$ is $\mathbb{S}_{D^{-}}$ generic over $V, \mathbf{d}$ is the unique branch through $\bigcap G$, and $f: D^{<\mathbb{N}} \rightarrow D$ is in $V$. Then there exists $n \in \mathbb{N}$ for which $\mathbf{d}(n)=f(\mathbf{d}\lceil n)$.

Proof. This follows from the fact that there are $\leq$-densely many trees $T \in S_{D}$ for which there exists $t \in D^{<\mathbb{N}}$ such that $t \frown(f(t))$ is ${ }^{-}$ comparable with every element of $T$.

Suppose now that $\mathrm{CH}$ holds in $V$, and fix a sequence $\left(D_{\beta}\right)_{\beta<\omega_{2}}$ of natural numbers that are at least two for which every such natural number appears cofinally often, as well as a countable support iteration $\left(\mathbb{P}_{\alpha}, \dot{\mathbb{Q}}_{\beta}\right)_{\alpha \leq \omega_{2}, \beta<\omega_{2}}$ such that $\vdash_{\mathbb{P}_{\beta}} \dot{\mathbb{Q}}_{\beta}=\left(\mathbb{S}_{D_{\beta}}\right)^{V^{\mathbb{P}} \beta}$ for all $\beta<\omega_{2}$.

As $\mathbb{P}_{\omega_{2}}$ has the Sacks property, it follows that $\operatorname{cof}(\mathcal{N})=\aleph_{1}$ in $V^{\mathbb{P} \omega_{2}}$ (see, for example, [Bla10, §11.5]). To see that $\chi\left(\mathbb{H}_{D^{\mathbb{N}}}\right)>\aleph_{1}$ in $V^{\mathbb{P}_{\omega_{2}}}$ for all natural numbers $D \geq 2$, note that if $\mathcal{F} \subseteq D^{D^{<\mathbb{N}}}$ is in $V^{\mathbb{P} \omega_{2}}$ and has cardinality at most $\aleph_{1}$ in $V^{\mathbb{P} \omega_{2}}$, then there exists $\alpha<\omega_{2}$ for which $\mathcal{F} \in V^{\mathbb{P}_{\alpha}}$. Fix $\beta \geq \alpha$ for which $D_{\beta}=D$, note that $D^{\mathbb{N}} \nsubseteq \bigcup_{f \in \mathcal{F}} \mathbf{D}_{f}$ in $V^{\mathbb{P}_{\beta+1}}$ by Lemma 6.12, and appeal to Proposition 6.3. 
Theorem $6.13\left(\mathrm{OGD}^{D}(\Gamma)\right)$. It is consistent that whenever $D$ is a finite set of cardinality at least two, $X$ is a Hausdorff space in $\Gamma$, and $H$ is an open $D$-dimensional dihypergraph on $X$, either $\chi(H) \leq \aleph_{0}$ or $\chi(H)>\operatorname{cof}(\mathcal{N})$.

Proof. It is sufficient to establish the consistency of $\chi\left(\mathbb{H}_{D^{\mathbb{N}}}\right)>\operatorname{cof}(\mathcal{N})$ for all finite sets $D$ of cardinality at least two, which follows from Theorem 6.11.

We finally establish a consistent upper bound as well.

Theorem 6.14. It is consistent that $\chi\left(\mathbb{H}_{D^{\mathbb{N}}}\right)<\mathfrak{d}$ for every finite set $D$ of cardinality at least three.

Proof. Given a natural number $D \geq 3$, let $P_{D}$ denote the set of pairs $(\mathbf{D}, f)$ with the property that $f: D^{<n} \rightarrow D$ for some $n \in \mathbb{N}, \mathbf{D} \subseteq \mathbf{D}_{f}$, and $\mathbf{c} \uparrow n \neq \mathbf{d} \uparrow n$ for all distinct $\mathbf{c}, \mathbf{d} \in \mathbf{D}$. Let $\leq$ denote the partial order on $P_{D}$ with respect to which $(\mathbf{C}, f) \leq(\mathbf{D}, g)$ if and only if $\mathbf{C} \subseteq \mathbf{D}$ and $f \sqsubseteq g$, and define $\mathbb{P}_{D}=\left(P_{D}, \leq\right)$.

Lemma 6.15. Suppose that $n \in \mathbb{N}$ and $f: D^{<n} \rightarrow D$. Then any two elements of $P_{D}$ of the form $(\mathbf{C}, f)$ and $(\mathbf{D}, f)$ are $\leq$-compatible.

Proof. Fix $m \geq n$ sufficiently large that $c \uparrow m \neq d \uparrow m$ for all distinct $c \in \mathbf{C}$ and $d \in \mathbf{D}$. As $D \geq 3$, there exists $g: D^{<m} \rightarrow D$ such that $\mathbf{C} \cup \mathbf{D} \subseteq \mathbf{D}_{g}$ and $f \sqsubseteq g$, in which case $(\mathbf{C} \cup \mathbf{D}, g)$ is a common $\leq$ extension of $(\mathbf{C}, f)$ and $(\mathbf{D}, f)$.

Fix a sequence $\left(D_{n}\right)_{n \in \mathbb{N}}$ of natural numbers that are at least three for which every such natural number appears cofinally often, and let $\mathbb{P}=(P, \leq)$ denote the finite support product of $\left(\mathbb{P}_{D_{n}}\right)_{n \in \mathbb{N}}$. Lemma 6.15 ensures that each of the partial orders $\mathbb{P}_{D}$ is $\sigma$-linked, thus so too is $\mathbb{P}$.

Lemma 6.16. Suppose that $D \geq 3$ is a natural number, $G$ is $\mathbb{P}$-generic over $V$, and $\mathbf{d} \in\left(D^{\mathbb{N}}\right)^{V}$. Then there exists $k \in \mathbb{N}$ such that $D_{k}=D$ and $\mathbf{d}(n) \neq f\left(\mathbf{d}\lceil n)\right.$ for all $n \in \mathbb{N}$, where $f=\bigcup\left\{f_{k} \mid\left(\mathbf{D}_{n}, f_{n}\right)_{n \in \mathbb{N}} \in G\right\}$.

Proof. This follows from the fact that there are $\leq$-densely many sequences $\left(\mathbf{D}_{n}, f_{n}\right)_{n \in \mathbb{N}} \in P$ for which there exists $k \in \mathbb{N}$ such that $D=D_{k}$ and $\mathbf{d} \in \mathbf{D}_{k}$.

Lemma 6.17. Suppose that $\dot{\mathbf{d}}$ is a $\mathbb{P}$-name for an element of $\mathbb{N}^{\mathbb{N}}$, $\left(k_{n}\right)_{n \in \mathbb{N}} \in \mathbb{N}^{\mathbb{N}}$, and $\left(f_{n}\right)_{n \in \mathbb{N}}$ is in the finite support product of $\left(D_{n}^{D_{n}^{<k_{n}}}\right)_{n \in \mathbb{N}}$. Then $\forall i \in \mathbb{N} \exists j \in \mathbb{N} \forall\left(\mathbf{D}_{n}, f_{n}\right)_{n \in \mathbb{N}} \in P\left(\mathbf{D}_{n}, f_{n}\right)_{n \in \mathbb{N}} \forall_{\mathbb{P}} \dot{\mathbf{d}}(i) \geq j$.

Proof. Suppose, towards a contradiction, that there exist $i \in \mathbb{N}$ and $\left(\mathbf{D}_{j, n}, f_{n}\right)_{n \in \mathbb{N}} \in P$ such that $\left(\mathbf{D}_{j, n}, f_{n}\right)_{n \in \mathbb{N}} \Vdash_{\mathbb{P}} \dot{\mathbf{d}}(i) \geq j$ for all $j \in \mathbb{N}$. 
By passing to a subsequence, we can assume that there are sequences $\left(I_{n}\right)_{n \in \mathbb{N}}$ of finite sets and $\left(\mathbf{d}_{i, j, n}\right)_{(i, j, n) \in I_{n} \times \mathbb{N} \times \mathbb{N}}$ of elements of $\mathbb{N}^{\mathbb{N}}$ such that $\mathbf{D}_{j, n}=\left\{\mathbf{d}_{i, j, n} \mid i \in I_{n}\right\}$ for all $j, n \in \mathbb{N}$ and $\mathbf{d}_{i, n}=\lim _{j \rightarrow \infty} \mathbf{d}_{i, j, n}$ exists for all $i, n \in \mathbb{N}$. Set $\mathbf{D}_{n}=\left\{\mathbf{d}_{i, n} \mid i \in I_{n}\right\}$. Then $\left(\mathbf{D}_{n}, f_{n}\right)_{n \in \mathbb{N}} \in P$, so there exist $k \in \mathbb{N}$ and an extension of $\left(\mathbf{D}_{n}, f_{n}\right)_{n \in \mathbb{N}} \in P$ for which $\left(\mathbf{D}_{n}^{\prime}, f_{n}^{\prime}\right)_{n \in \mathbb{N}} \Vdash_{\mathbb{P}} \dot{\mathbf{d}}(i)=k$. As $\left(\mathbf{D}_{j, n}, f_{n}^{\prime}\right)_{n \in \mathbb{N}} \in P$ for all sufficiently large $j \in \mathbb{N}$, Lemma 6.15 ensures that $\left(\mathbf{D}_{j, n}, f_{n}\right)_{n \in \mathbb{N}}$ and $\left(\mathbf{D}_{n}^{\prime}, f_{n}^{\prime}\right)_{n \in \mathbb{N}}$ are compatible for all sufficiently large $j>k$, the desired contradiction. $\square$

Suppose now that $\mathfrak{d}>\aleph_{1}$ in $V$, and fix a finite support iteration $\left(\mathbb{P}_{\alpha}, \dot{\mathbb{Q}}_{\beta}\right)_{\alpha \leq \omega_{1}, \beta<\omega_{1}}$ such that $\Vdash_{\mathbb{P}_{\beta}} \dot{\mathbb{Q}}_{\beta}=\mathbb{P}^{V^{\mathbb{P}_{\beta}}}$ for all $\beta<\omega_{1}$.

Proposition 6.3 and Lemma 6.16 easily imply that $\chi\left(\mathbb{H}_{D^{\mathbb{N}}}\right)=\aleph_{1}$ in $V^{\mathbb{P}_{\omega_{1}}}$. To see that $\mathfrak{d}>\aleph_{1}$ in $V^{\mathbb{P} \omega_{1}}$, note that if $\left(\dot{\mathbf{d}}_{\alpha}\right)_{\alpha<\omega_{1}} \in V$ is a sequence of $\mathbb{P}_{\omega_{1}}$-names for elements of $\mathbb{N}^{\mathbb{N}}$, then Lemma 6.17 yields a sequence $\left(\mathbf{d}_{\alpha}\right)_{\alpha<\omega_{1}} \in V$ of elements of $\mathbb{N}^{\mathbb{N}}$ such that $\Vdash_{\mathbb{P}_{\omega_{1}}} \dot{\mathbf{d}}_{\alpha} \leq{ }^{*} \mathbf{d}_{\alpha}$ for all $\alpha<\omega_{1}$. The fact that $\mathfrak{d}>\aleph_{1}$ in $V$ then yields $\mathbf{d} \in\left(\mathbb{N}^{\mathbb{N}}\right)^{V}$ such that $\mathbf{d} \not \mathbb{Z}^{*} \mathbf{d}_{\alpha}$ for all $\alpha<\omega_{1}$, so $\Vdash_{\mathbb{P}_{\omega_{1}}} \mathbf{d} \not \mathbb{L}^{*} \dot{\mathbf{d}}_{\alpha}$ for all $\alpha<\omega_{1}$.

\section{REFERENCES}

[Bla10] Andreas Blass, Combinatorial cardinal characteristics of the continuum, Handbook of set theory. Vols. 1, 2, 3, Springer, Dordrecht, 2010, pp. 395489. MR 2768685

[Bre03] Jörg Brendle, Evasion and prediction. III. Constant prediction and dominating reals, J. Math. Soc. Japan 55 (2003), no. 1, 101-115. MR 1939187

[Dav64] Morton Davis, Infinite games of perfect information, Advances in game theory, Princeton Univ. Press, Princeton, N.J., 1964, pp. 85-101. MR 0170727

[Fen93] Q. Feng, Homogeneity for open partitions of pairs of reals, Trans. Amer. Math. Soc. 339 (1993), no. 2, 659-684. MR 1113695 (93m:03081)

[Hur28] Witold Hurewicz, Relativ perfekte teile von punktmengen und mengen (a), Fund. Math. 12 (1928), 78-109.

[JR82] J.E. Jayne and C.A. Rogers, First level Borel functions and isomorphisms, J. Math. Pures Appl. (9) 61 (1982), no. 2, 177-205. MR 673304 (84a:54072)

[Kam00] Shizuo Kamo, Cardinal invariants associated with predictors, Logic Colloquium '98 (Prague), Lect. Notes Log., vol. 13, Assoc. Symbol. Logic, Urbana, IL, 2000, pp. 280-295. MR 1743265

[Kec77] Alexander S. Kechris, On a notion of smallness for subsets of the Baire space, Trans. Amer. Math. Soc. 229 (1977), 191-207. MR 0450070

[KLW87] A. S. Kechris, A. Louveau, and W. H. Woodin, The structure of $\sigma$-ideals of compact sets, Trans. Amer. Math. Soc. 301 (1987), no. 1, 263-288. MR 879573

[Lec13] Dominique Lecomte, Potential Wadge classes, Mem. Amer. Math. Soc. 221 (2013), no. 1038, vi+83. MR 3057834 
[LSR87] A. Louveau and J. Saint-Raymond, Borel classes and closed games: Wadge-type and Hurewicz-type results, Trans. Amer. Math. Soc. 304 (1987), no. 2, 431-467. MR 911079

[LZ14] D. Lecomte and M. Zeleny, Baire-class $\xi$ colorings: the first three levels, Trans. Amer. Math. Soc. 366 (2014), no. 5, 2345-2373. MR 3165641

[Sol98] S. Solecki, Decomposing Borel sets and functions and the structure of Baire class 1 functions, J. Amer. Math. Soc. 11 (1998), no. 3, 521-550. MR 1606843 (99h:26010)

[Sou17] M. Ya. Souslin, Sur une définition des ensembles mesurables B sans nombres transfinis, C. R. Math. Acad. Sci. Paris 164 (1917), 88-91.

[SR75] Jean Saint-Raymond, Approximation des sous-ensembles analytiques par l'intérieur, C. R. Acad. Sci. Paris Sér. A-B 281 (1975), no. 2-3, Aii, A85-A87. MR 0377840

[SR78] , La structure borélienne d'Effros est-elle standard?, Fund. Math. 100 (1978), no. 3, 201-210. MR 509546

[Wad12] William W. Wadge, Early investigations of the degrees of Borel sets, Wadge degrees and projective ordinals. The Cabal Seminar. Volume II, Lect. Notes Log., vol. 37, Assoc. Symbol. Logic, La Jolla, CA, 2012, pp. 166-195. MR 2906999

Raphaël Carroy, Kurt Gödel Research Center for Mathematical Logic, Universität Wien, Währinger Strasse 25, 1090 Wien, Austria

E-mail address: raphael.carroy@univie.ac.at

$U R L:$ http://www.logique.jussieu.fr/ ${ }^{\sim}$ carroy/indexeng.html

Benjamin D. Miller, Kurt Gödel Research Center for MathematiCal Logic, Universität Wien, Währinger Strasse 25, 1090 Wien, AusTRIA

E-mail address: benjamin.miller@univie.ac.at

URL: http://www.logic.univie.ac.at/benjamin.miller

Dániel T. Soukup, Kurt Gödel Research Center for Mathematical Logic, Universität Wien, Währinger Strasse 25, 1090 Wien, Austria

E-mail address: daniel.soukup@univie.ac.at

URL: http://www.logic.univie.ac.at/ soukupd73 